\title{
Lessons Learned from Numerical Simulations of the F-16XL Aircraft at Flight Conditions
}

\author{
Arthur Rizzi* \\ Royal Institute of Technology KTH, SE-10044 Stockholm, Sweden \\ Adam Jirásek ${ }^{\dagger}$ \\ Swedish Defence Research Agency FOI, SE-16490 Stockholm, Sweden \\ John E. Lamar \\ Lamar Engineering Consultanship, Newport News, VA 23608 USA \\ Simone Crippa ${ }^{\S}$ \\ Royal Institute of Technology KTH, SE-10044 Stockholm, Sweden
}

Kenneth J. Badcock

University of Liverpool, Liverpool L693GH, UK

Okko J. Boelens $\|$

National Aerospace Laboratory NLR, 1059 CM Amsterdam, The Netherlands

\begin{abstract}
Nine groups participating in the CAWAPI project have contributed steady and unsteady viscous simulations of a full-scale, semi-span model of the F-16XL aircraft. Three different categories of flight Reynolds/Mach number combinations were computed and compared with flight-test measurements for the purpose of code validation and improved understanding of the flight physics. Steady-state simulations are done with several turbulence models of different complexity with no topology information
\end{abstract}

${ }^{*}$ Professor, Department of Aeronautical \& Vehicle Engineering, AIAA Associate Fellow

${ }^{\dagger}$ Research Engineer, Division of Systems Technology, AIAA Member

${ }^{\ddagger}$ CAWAP Principal Investigator, NASA-LaRC Retired, AIAA Associate Fellow

$\S$ Research assistant, Department of Aeronautical \& Vehicle Engineering, AIAA Student Member

`Professor of Computational Aerodynamics, Department of Engineering, AIAA Member

${ }^{\| R \& D}$ Engineer, Applied Computational Fluid Dynamics, Department of Flight Physics and Loads, Aerospace Vehicles Division, AIAA member 
required and which overcome Boussinesq-assumption problems in vortical flows. Detached-eddy simulation (DES) and its successor delayed detachededdy simulation (DDES) have been used to compute the time accurate flow development. Common structured and unstructured grids as well as individually-adapted unstructured grids were used. Although discrepancies are observed in the comparisons, overall reasonable agreement is demonstrated for surface pressure distribution, local skin friction and boundary velocity profiles at subsonic speeds. The physical modeling, steady or unsteady, and the grid resolution both contribute to the discrepancies observed in the comparisons with flight data, but at this time it cannot be determined how much each part contributes to the whole. Overall it can be said that the technology readiness of CFD-simulation technology for the study of vehicle performance has matured since 2001 such that it can be used today with a reasonable level of confidence for complex configurations

\section{Nomenclature}

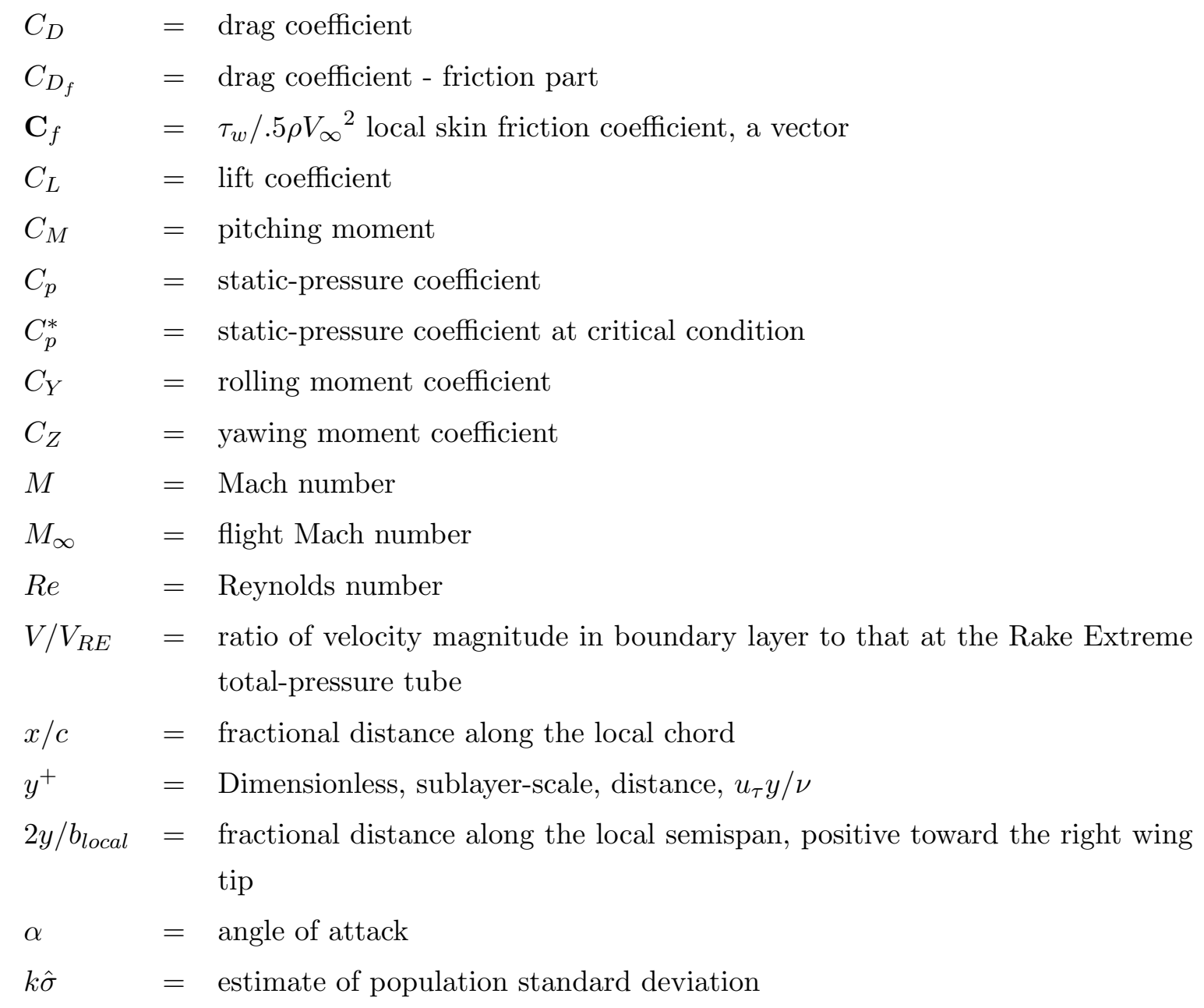




$\begin{array}{ll}\lambda_{2} & =\text { second eiganvalue of } \mathbf{S}-\Omega \text { tensor } \\ \hat{\mu} & =\text { estimate of population mean }\end{array}$

\section{Organizations}

$\begin{array}{ll}\text { Boeing } & \text { Boeing-Phantom Works-St.Louis, MO USA } \\ \text { EADS-MAS }= & \text { European Aeronautics and Defence Company - Military Aircraft Sys- } \\ & \text { tems, Germany } \\ \text { FOI } & \text { Swedish Defence Research Agency, Sweden } \\ \text { KTH } & \text { Royal Institute of Technology, Sweden } \\ \text { LM Aero } & \text { Lockheed Martin Aeronautics Company, TX USA } \\ \text { NASA } & \text { NASA-LaRC-Hampton, National Aeronautics and Space Administra- } \\ \text { NLR } & =\text { Nion, VA USA } \\ \text { ULiv } & =\text { University of Liverpool, UK } \\ \text { UTSimC } & \text { University of Tennessee at Chattanooga, TN USA } \\ \text { USAFA } & =\text { United States Air Force Academy, CO USA }\end{array}$

\section{Introduction}

The original NASA document ${ }^{1}$ together with the first of the preceding articles, ${ }^{2}$ in this Special Section (so-called companion papers) have introduced the problem that the AVT-113 task group studied, that of increasing the Technology Readiness Level (TRL) of the CFD solvers by establishing best practices with the solvers as applied to the F-16XL aircraft at a number of flight conditions. Another one of the preceding articles ${ }^{3}$ reported how the grids were established for each solver type (structured, unstructured common or tailored) or with a particular solver studied, and the remaining companion papers ${ }^{4-6}$ in this series presented analyses and representative results for each solver type. The current article draws from some of these companion papers as well as the base papers, ${ }^{7-16}$ presented at two Special Sessions of the AIAA ASM Conference in January 2007, along with new analysis and summaries, not previously available, to put into perspective what was accomplished by the Cranked Arrow Wing Aerodynamics Project International (CAWAPI) facet of the AVT-113 task group. Moreover, CFD comparisons are also offered with those reported in the basic document ${ }^{1}$ which was used to launch this study.

This paper compares cumulatively all the computed results with the flight-test measurements, makes some overall observations about these comparisons, discusses some likely causes for discrepancies, and where possible draws tentative conclusions and tries to iden- 
tify lessons learned in order to take a step forward towards establishing some best practices for this class of problem. Similar cumulative-comparison activities have been done before, notably among them, the series of AIAA Drag Prediction Workshops. What we do here differs in that it is a fighter configuration, a flying aircraft, and the comparisons are done against flight-test data not windtunnel measurements, which makes it rather unique. Furthermore the objective is not the accurate prediction of say total drag counts, but instead is the prediction of complex vortical-flow phenomena that strongly impact the flying qualities of a fighter aircraft because the interaction of vortices over such an aircraft determines its stability and control characteristics.

The lessons learned in this five-year study are reported here by first reviewing the impact of the grid on the CFD solvers and the ensuing solutions, then the predictive capabilities themselves of the solvers for selected dominant vortical- and transonic-flow flight conditions, along with any deficiencies are noted. The flight condition (FC) at the highest angle of attack (FC25) is discussed using three sets of representative results. This case showed significant unsteady flow. For FC19, results are compared for the prediction of skin friction and for boundary-layer profiles in FC7. Next, a wide ranging effort to explain significant discrepancies between measurements and computations for the transonic flight condition (FC70) is described. A high-level overall assessment is then made by first comparing progress achieved since the NASA publication ${ }^{1}$ from 2001, and secondly by quoting comments made by industry participants regarding the value of their participation in the study. Finally, conclusions are stated.

\section{Overview of Codes and Comparisons}

Table 1 lists the ten solvers utilized in CAWAPI along with a brief description of relevant information about each, including the models employed, whether steady or unsteady flow was computed and the grid type used. The flight conditions computed by each partner are also listed. The geometry used during this exercise is a detailed model of the F-16XL geometry. Details on both geometry and grids can be found in Obara and Lamar ${ }^{17}$ and Boelens et al. ${ }^{3}$ More details on the solvers can be found in references. ${ }^{4-6}$

Note that all turbulence models integrate to the wall with the exception of LM Aero, which used wall functions. The rows in the table are grouped by the grid type used. The first group used a common multiblock grid. ${ }^{4}$ The second group used a common unstructured grid. ${ }^{5}$ Finally, the third group used tailored grids, referred to in this paper as trimmed grids. ${ }^{6}$

The flight conditions are defined in detail in Obara and Lamar. ${ }^{18}$ In this paper the conditions computed are split into two groups. The first (FC 7, 19, 46, 50 and 51) are dominated by strong vortices, and have low subsonic freestream Mach numbers, high incidence and 
flight Reynolds numbers. The second group (FC70) is at high subsonic freestream Mach numbers and a relatively low incidence, and features shock waves and a weak vortex. The comparisons are made separately for these two groups of conditions.

Table 1. Overview of ten solutions generated by the nine participating organizations.

\begin{tabular}{|c|c|c|c|c|}
\hline Contributor & Grid type & Turbulence models & Numerics $^{\dagger}$ & FC \\
\hline $\mathrm{NLR}^{7,19}$ & $\begin{array}{l}\text { common } \\
\text { structured }\end{array}$ & $\begin{array}{l}k-\omega+\text { vorticity correc- } \\
\text { tions }\end{array}$ & CC, central & $\begin{array}{ll}\text { FC07, } & \text { FC19, } \\
\text { FC25, } & \text { FC46, } \\
\text { FC50, } & \text { FC51, } \\
\text { FC70 } & \end{array}$ \\
\hline $\begin{array}{l}\text { University of } \\
\text { Liverpool }^{9}\end{array}$ & $\begin{array}{l}\text { common } \\
\text { structured }\end{array}$ & $\begin{array}{l}k-\omega \text { TNT + vorticity cor- } \\
\text { rections }\end{array}$ & $\begin{array}{l}\text { CC, Osher upwind, } \\
\text { Roe flux-vector split- } \\
\text { ting }\end{array}$ & $\begin{array}{ll}\text { FC07, } & \text { FC19, } \\
\text { FC25, } & \text { FC46, } \\
\text { FC70 } & \end{array}$ \\
\hline $\begin{array}{l}\text { NASA- } \\
\text { LARC }^{10}\end{array}$ & $\begin{array}{l}\text { common } \\
\text { structured }\end{array}$ & $\begin{array}{l}k-\omega, \text { SZL EARSM, Giri- } \\
\text { maji EARSM }\end{array}$ & $\begin{array}{l}\text { CC, Roe flux-vector } \\
\text { splitting, implicit } 3 \\
\text { factor scheme, explicit } \\
\text { - DTS }\end{array}$ & $\begin{array}{ll}\text { FC07, } & \text { FC19, } \\
\text { FC25, } & \text { FC46, } \\
\text { FC50, } & \text { FC51, } \\
\text { FC70 } & \end{array}$ \\
\hline USAFA $^{15}$ & $\begin{array}{l}\text { common un- } \\
\text { structured }\end{array}$ & $\begin{array}{l}\text { SARC-DES } \\
\text { DDES }\end{array}$ & $\begin{array}{l}\text { CC, Godunov type } \\
\text { with least square ap- } \\
\text { proximation, implicit } \\
\text { with Newton method }\end{array}$ & $\begin{array}{ll}\text { FC07, } & \text { FC19 }{ }^{\star} \\
\text { FC25 } & \text { FC46 } \\
\text { FC50^} & \text { FC51 } \\
\text { FC70 } & \end{array}$ \\
\hline $\mathrm{KTH} / \mathrm{FOI}^{11}$ & $\begin{array}{l}\text { common and } \\
\text { trimmed un- } \\
\text { structured }\end{array}$ & $\begin{array}{l}\text { SA, Hellsten EARSM } \\
k-\omega \ddagger \text { Hellsten EARSM } \\
k-\omega+\text { rotational cor- } \\
\text { rections, DES, Hybrid } \\
\text { RANS-LES }\end{array}$ & $\mathrm{NC}$, central & $\begin{array}{ll}\text { FC07, } & \text { FC19, } \\
\text { FC25, } & \text { FC46, } \\
\text { FC50, } & \text { FC51, } \\
\text { FC70 (Euler, } \\
\text { RANS) }\end{array}$ \\
\hline $\begin{array}{l}\text { NASA- } \\
\text { LARC }^{14}\end{array}$ & $\begin{array}{l}\text { common un- } \\
\text { structured }\end{array}$ & linear $k$ - $\epsilon \ddagger$ non-linear $k$ - $\epsilon$ & $\begin{array}{l}\text { CC, Roe FDS, implicit } \\
\text { scheme }\end{array}$ & $\begin{array}{ll}\text { FC07, } & \text { FC19, } \\
\text { FC25, } & \text { FC46, } \\
\text { FC50, } & \text { FC51, } \\
\text { FC70 } & \end{array}$ \\
\hline $\begin{array}{l}\text { EADS- } \\
\text { MAS }^{12}\end{array}$ & $\begin{array}{l}\text { trimmed un- } \\
\text { structured }\end{array}$ & SA & $\begin{array}{l}\text { NC, AUSMDV, back- } \\
\text { ward Euler implicit + } \\
\text { LU-SGS }\end{array}$ & $\begin{array}{ll}\text { FC07, } & \text { FC19, } \\
\text { FC25, } & \text { FC46, } \\
\text { FC50, } & \text { FC51, } \\
\text { FC70 } & \end{array}$ \\
\hline $\begin{array}{l}\text { UT SimCen- } \\
\text { ter }^{13}\end{array}$ & $\begin{array}{l}\text { trimmed un- } \\
\text { structured }\end{array}$ & $k-\omega k-\epsilon$ hybrid & $\begin{array}{l}\text { NC, Roe flux-vector } \\
\text { splitting, point im- } \\
\text { plicit }\end{array}$ & $\begin{array}{ll}\text { FC07, } & \text { FC19, } \\
\text { FC25, } & \text { FC46, } \\
\text { FC50, } & \text { FC51, } \\
\text { FC70 } & \end{array}$ \\
\hline Boeing ${ }^{8}$ & $\begin{array}{l}\text { trimmed un- } \\
\text { structured }\end{array}$ & $\begin{array}{l}\text { SA, Menter SST, SST- } \\
\text { LESb }\end{array}$ & CC, HLLE, FDS & $\begin{array}{ll}\text { FC07, } & \text { FC19, } \\
\text { FC25, } & \text { FC46, } \\
\text { FC70 } & \end{array}$ \\
\hline LM Aero ${ }^{16}$ & $\begin{array}{l}\text { trimmed un- } \\
\text { structured }\end{array}$ & $k-k l$ & $\begin{array}{l}\text { Roe approximate Rie- } \\
\text { mann solver, implicit } \\
\text { ILU }\end{array}$ & $\begin{array}{l}\text { FC07, FC19, } \\
\text { FC70 }\end{array}$ \\
\hline
\end{tabular}

$\ddagger$ Turbulence model used in common comparisons.

$\dagger$ Numerical method cell-centered (CC) or node-centered (NC).

${ }^{\star}$ Flight condition calculated as unsteady. 


\section{Low-Speed Flow Cases}

\section{A. Unsteady Flow at High $\alpha-$ FC25}

\section{Overview}

The conditions for FC25 are $M_{\infty}=0.242, \alpha=19.8^{\circ}, R e=32.22 \times 10^{6}$. FC25 has the largest angle of attack and thus better defined vortical flow phenomena are expected over the upper surface, perhaps even vortex breakdown over the aft portion of the aircraft. Large scale flow unsteadiness is also associated with such phenomena. For this flight condition surface pressure measurements are available.

\section{Force/Moments Comparison}

Table 2 shows the comparison of of the seven reported sets of forces and moments.

Table 2. Flight condition FC25, force/moment coefficients. Details about grids ${ }^{3}$ and solvers ${ }^{4-16}$ used are given in the references, also refer to Table 1.

\begin{tabular}{lccccccc}
\hline \hline Coefficient & NLR & KTH/FOI & USAFA & NASA - unstr & EADS $^{\dagger}$ & UT SimC & Boeing \\
\hline$C_{L}$ & 0.77328 & 0.79789 & 0.78402 & 0.80199 & 0.82795 & 0.76553 & 0.75560 \\
$C_{D}$ & 0.45088 & 0.44800 & 0.40537 & 0.49923 & 0.44577 & 0.59244 & 0.52836 \\
$C_{D_{f}}$ & 0.01270 & 0.01060 & - & 0.01989 & - & 0.01373 & 0.01030 \\
$C_{M}$ & 0.02525 & 0.01061 & 0.01645 & 0.01653 & -0.00492 & 0.00707 & 0.02228 \\
\hline \hline
\end{tabular}

$\dagger$ Data without inlet and nozzle surface

The statistical analysis of the data from Table 2 is shown in Table 3 listing the population mean $\hat{\mu}$ and the value $k \hat{\sigma}$ to add/subtract to reach the upper/lower limit that defines the population of "identical" solutions (see Appendix). One of the seven solutions is found to be an outlier. In comparison with the data sets for the other flight conditions (FC19, FC7, FC50, FC51 and FC70), FC25 has a half-width $k \hat{\sigma}$ of the upper-lower band of identical solutions that is approximately twice that of all the other flight conditions. Thus this data set possesses the largest disparity.

Table 3. Flight condition FC25, force/moment coefficients - statistical analysis. Details about grids $^{3}$ and solvers ${ }^{4-16}$ used are given in the references, also refer to Table 1.

\begin{tabular}{lcccc}
\hline \hline Coefficient & $\hat{\mu}$ & $k \hat{\sigma}$ & \# identical solutions & \# outliers \\
\hline$C_{L}$ & 0.78402 & 0.04307 & 6 & 1 \\
$C_{D}$ & 0.45088 & 0.12346 & 6 & 1 \\
$C_{D_{f}}$ & 0.01270 & 0.00687 & 4 & 1 \\
$C_{M}$ & 0.01645 & 0.01858 & 6 & 1 \\
\hline \hline
\end{tabular}




\section{Vortical Flow Features}

Figure 1 presents sectional spanwise contours of iso-total-pressure, skin friction lines and flight tuft patterns. The contour plots indicate the presence and location of the inner

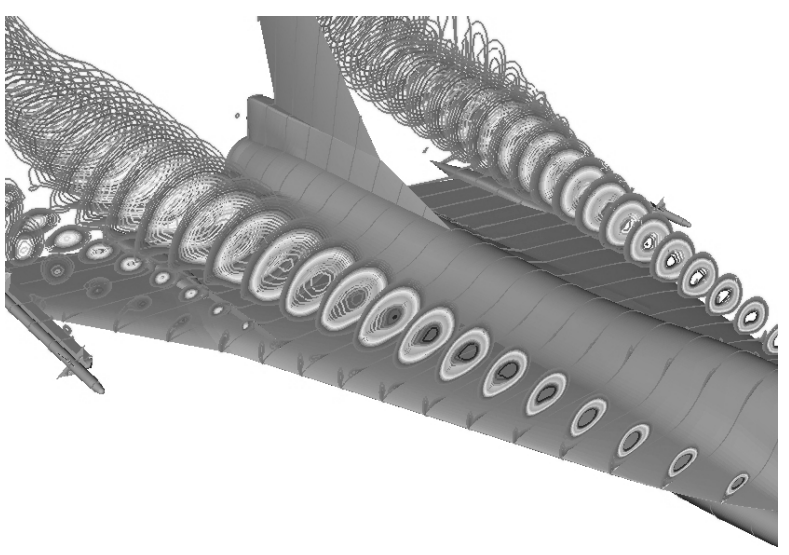

(a) Sectional spanwise iso-total-pressure contours.

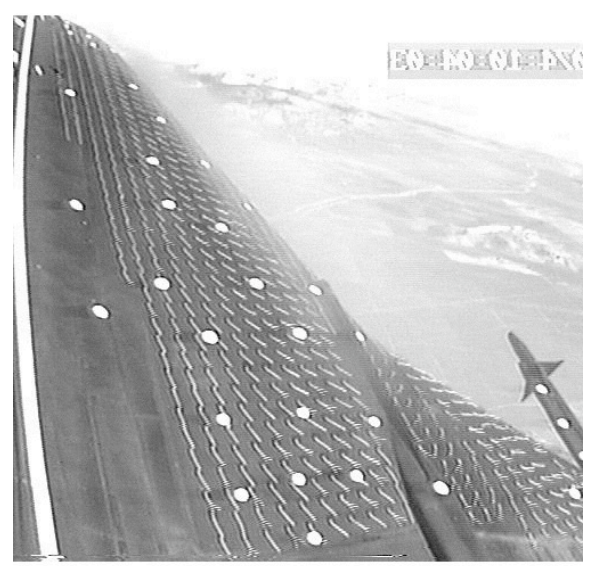

(c) Flight tuft photo from vertical-tail topleft camera mirrored about the vertical axis of the photo. ${ }^{1}$

Figure 1. Vortical-flow features over upper surface for FC25. Details about grids ${ }^{3}$ and solvers $^{4-16}$ used are given in the references, also refer to Table 1.

wing primary and secondary vortices, the outer wing primary and secondary vortices. The convergence-divergence of skin friction lines indicates the occurrence and location of primary or secondary vortex separation-reattachment, respectively. Figure 1(b) suggests that the separation layer on the fuselage is now drawn outboard towards the actuator pod where it may merge with the vortical flow around that structure. There are striking overall sim- 
ilarities with the in-flight photographs of the tufts shown in Figure 1(c). Similar behavior is observed for the inward flow to the airdam on both the inner and outer wings and the marked flow division that occurs on the outer wing. Differences are seen for the inner wing spanwise flow extending toward the fuselage, especially near the trailing edge.

\section{4. $C_{p}$ Comparisons along BL Sections}

A down-select of the ten solutions makes the FC25 $C_{p}$ comparison with flight data more tractable. One solution from each type of grid was selected, namely NLR for the standard structured grid, EADS for the tailored unstructured grid, and USAFA for the standard unstructured grid. Keep in mind that the first two results are steady-state RANS solutions whereas the third (USAFA) is a time-accurate DES solution. ${ }^{15}$ These three results have been cross-plotted with the flight data and the previous CFL3D solutions, ${ }^{1}$ and their comparisons are now shown. The time-averaged value (mean) is plotted for the unsteady USAFA solution. In their companion paper Goertz et $a .^{5}{ }^{5}$ report significant unsteadiness in their solutions located over the rear of the wing, close to the wing tip, especially at BL 184.5.

Figure 2 shows comparisons of the computed and flight measured surface pressure coefficients $C_{p}$ plotted along the butt lines, the first four along the inner wing and the last two along the outer wing where we expect strong interactions. The first four butt lines (BL55, BL70, BL80 and BL95) show that the pressures associated with inner wing primary and secondary vortices are being resolved. The leading edge vortex forms in the vicinity of BL55 and the measured values show a primary-vortex peak as well as a secondary-vortex peak, as do some, but not all, of the computed results. The fall off in primary suction peak from BL55 to BL95 is associated with the center of the vortex system moving farther from the surface the further the system travels downstream. This feature is caused by the system capturing more leading-edge shed vorticity, as it traverses the wing, yielding an enlarged cross-section. Morton et al. ${ }^{15}$ present similar inner BL plots in which they plot the instantaneous values of minimum and maximum $C_{p}$ that forms a band around the time-averaged mean values. Over the inner wing this band is very thin, indicating that the flow here is steady.

At BL153.5 and BL184.5 the predicted and measured pressures demonstrate that there are strong interactions between the vortices shed from the inner wing, actuator pod, airdam and crank. At BL153.5, just outboard of the juncture of these vortices, the computed and measured results show a suction peak for the outer wing primary vortex near $15 \%$ chord and a smaller peak near $75 \%$ chord from the combination of actuator pod/airdam/inner wing secondary vortices; however, the predictions for the second peak have something of a spread in the peak value and its location. At BL184.5 the measured peak value for the outer-wing primary vortex is much reduced and its location occurs closer the leading-edge, near $10 \%$ chord. A second, smaller peak is predicted to occur at this BL near mid-chord and 
is primarily associated with the inner-wing secondary vortex (See Fig. 1(b)). Note that the flow in the vicinity of the wing tip is made even more complicated because of the vortex wake off of the missile fins, especially at this $\alpha$. For these two BL locations on the outer wing, the plot in Morton et al. ${ }^{15}$ now shows bands between the minimum and maximum instantaneous $C_{p}$ values that are very broad and encompass the measured data. This indicates that the flow over the outboard wing section has substantial unsteadiness. It also suggests that the USAFA mean value plotted in Fig. 2(e) and 2(f) is a more accurate estimate of the unsteady pressure distribution than the other Reynolds-averaged steady-state results.

From the comparisons presented, the solver used by the USAFA with the DES turbulence model produced the best overall agreement with the flight data, indicating that for this $\alpha$, modeling the large-scale unsteadiness in a time accurate mode is a key to improved predictability. All of the new solvers showed superior agreement with data in comparison to that documented in Lamar et al. ${ }^{1}$ for the CFL3D solver with an order of magnitude fewer grid points used and larger $y^{+}$value.

\section{B. Skin Friction Coefficient - FC19}

\section{Overview}

The conditions for FC19 are $M_{\infty}=0.36, \alpha=11.85^{\circ}, R e=46.8 \times 10^{6}$. FC19 is also a case of fully developed vortical flow over the upper surface. For this flight condition skin friction measurements are available.

\section{Vortical Flow Features}

Figure 3 shows sectional spanwise contours of iso-total-pressure and skin friction lines. The contour plots indicate the presence and location of the inner-wing primary and secondary vortices, the outer-wing primary and secondary vortices. Note the presence of small fuselage vortices which were not present for FC25 (see Figure 1).

\section{Skin Friction Comparison}

Figure 4 compares the computed and measured values of the skin friction coefficient $\mathbf{C}_{f}$ at fuselage station (FS) 330, FS330. In general the skin friction coefficient $\mathbf{C}_{f}$ is a vector quantity. The measurements were made with modified Preston tubes which pointed into the local flow direction - which varied across the FS330. The fact that the local skin friction can be correlated with the delta-p between the static and total pressure is the basis of these measurements, but these are a magnitude measurement. The comparison is made with the magnitude of the computed vector quantity. The location FS330 is in the general vicinity 


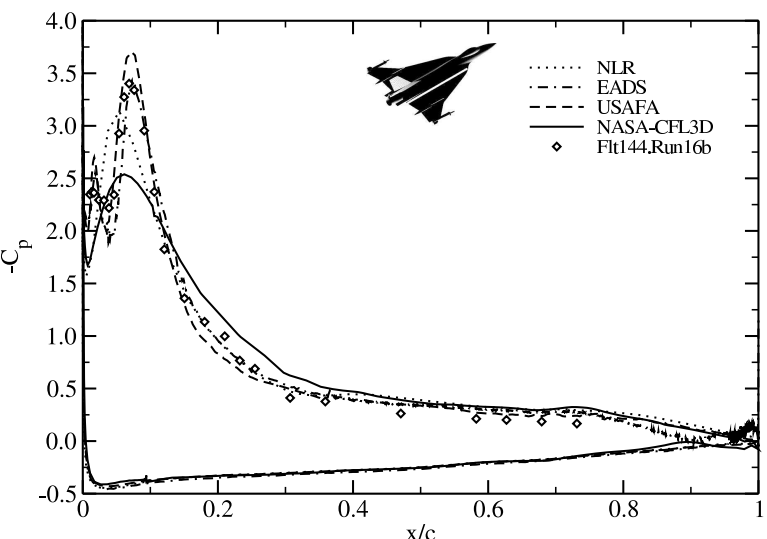

(a) $\mathbf{B L} 55$

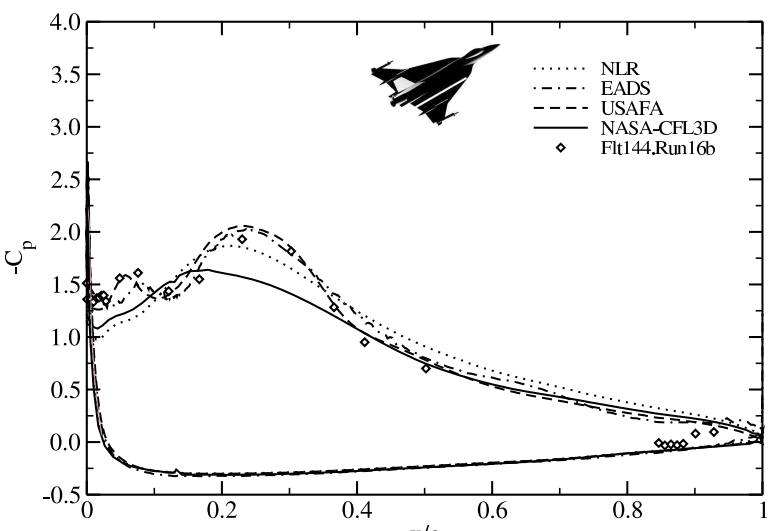

(c) $\mathbf{B L}^{\mathrm{x} / \mathrm{c}} \mathrm{80}$

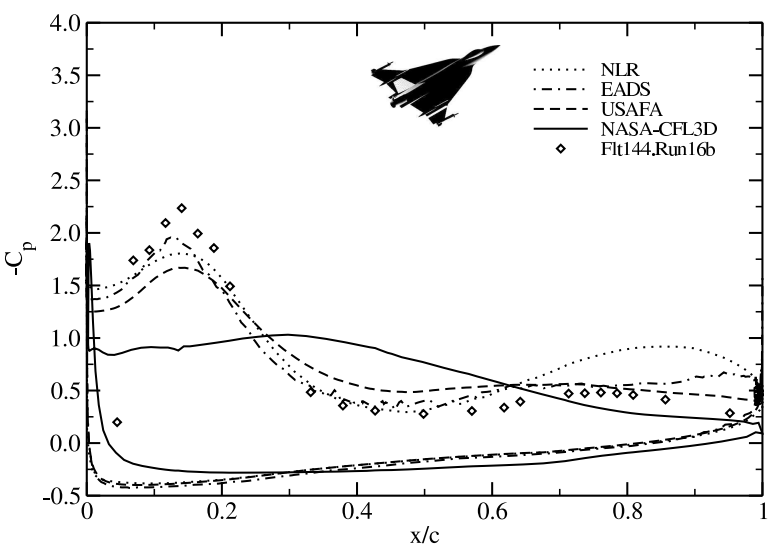

(e) BL153.5

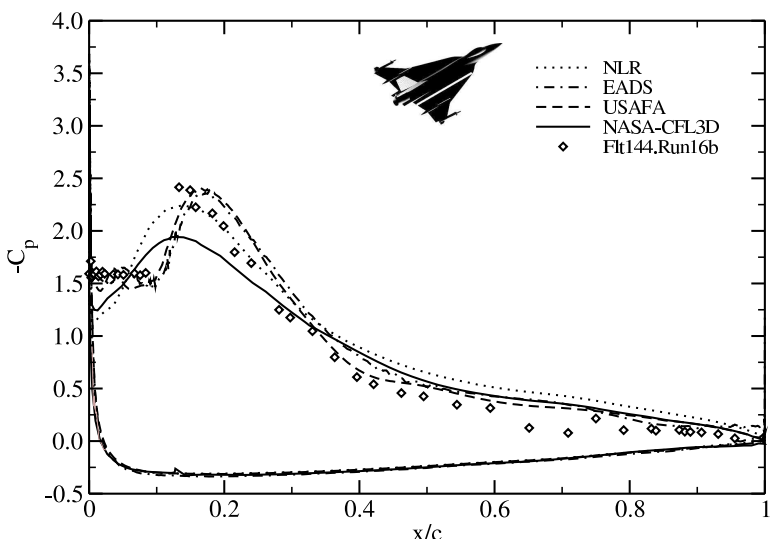

(b) BL70

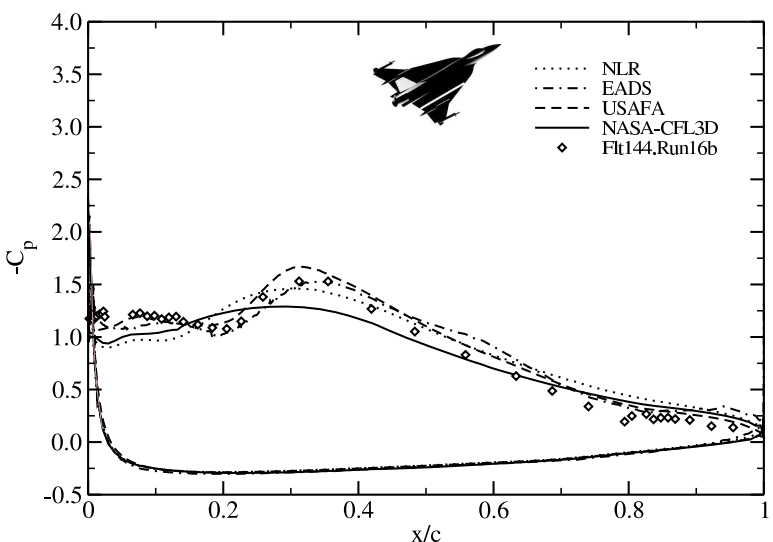

(d) BL95

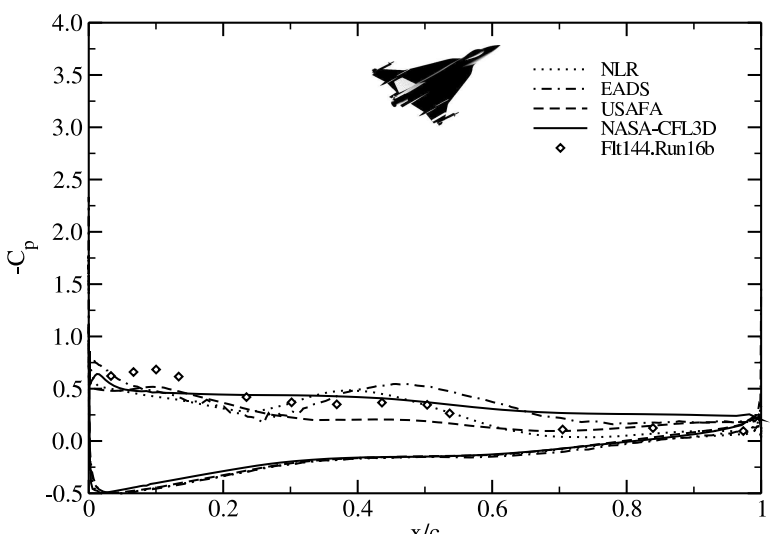

(f) $\operatorname{BL184.5}$

Figure 2. Chordwise comparison for FC25 of pressure coefficient. Details about grids ${ }^{3}$ and solvers ${ }^{4-16}$ used are given in the references, also refer to Table 1. 


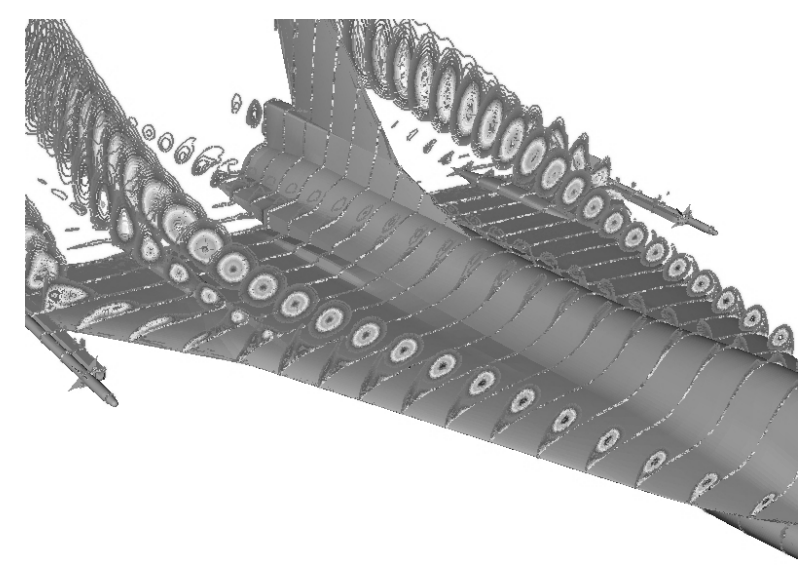

(a) Sectional spanwise iso-total-pressure contours.

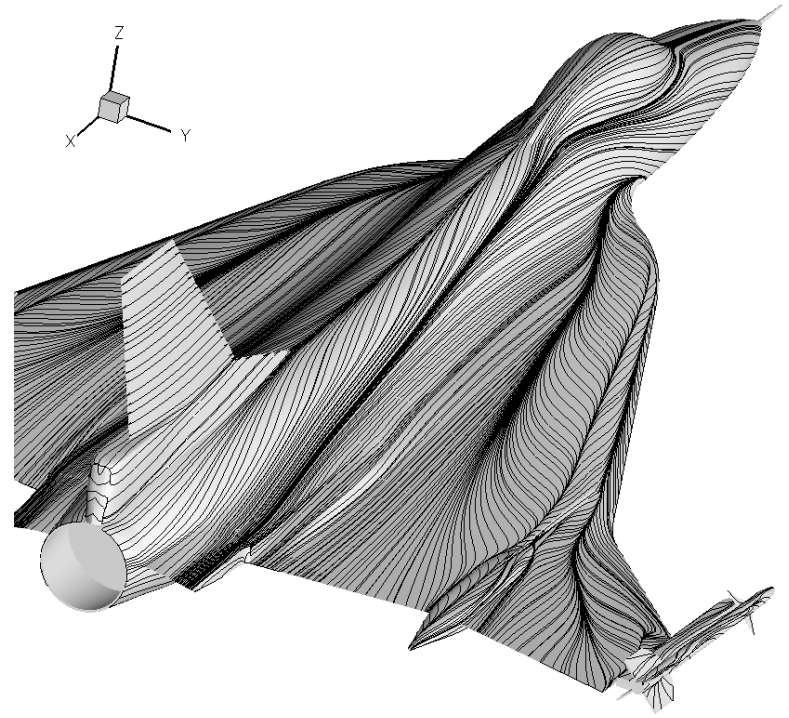

(b) Skin friction lines.

Figure 3. Vortical-flow features over upper surface for FC19. Details about grids ${ }^{3}$ and solvers $^{4-16}$ used are given in the references, also refer to Table 1.

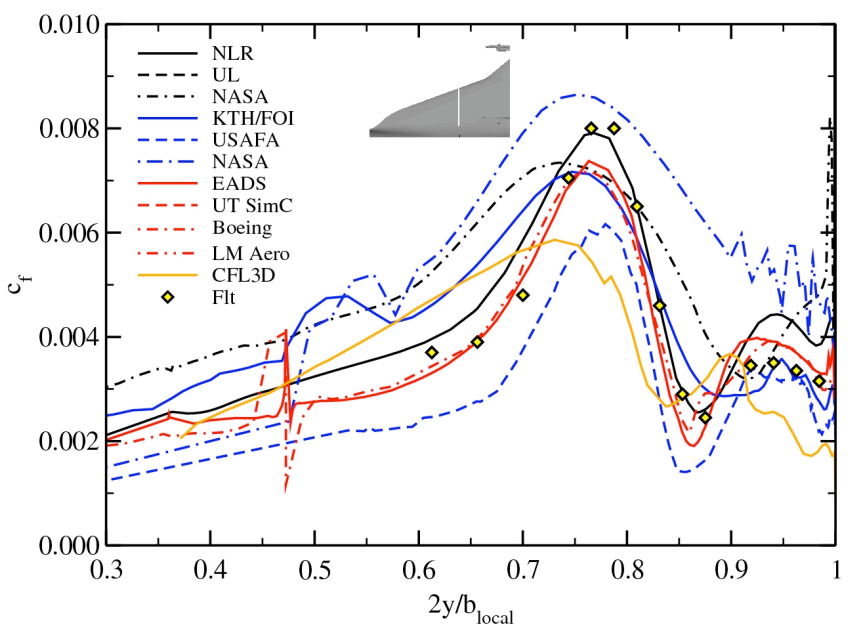

\section{Color coding: Black - Common structured mesh \\ Blue - Common unstructured mesh \\ Red - Trimmed unstructured mesh}

Figure 4. Spanwise comparison (FS330) for FC19 of the magnitude of the skin friction coefficient $\mathrm{C}_{f}$. Details about grids ${ }^{3}$ and solvers ${ }^{4-16}$ used are given in the references, also refer to Table 1. 
of the boundary-layer rakes discussed below. The measured values show two peaks in $C_{f}$, the highest under the primary inner-wing vortex and the lower one under the secondary inner-wing vortex. The computed results of KTH/FOI and NASA-U shows three peaks, the inboard one occurs at a location where there are no measurements that could confirm it. USAFA results, which were obtained on the same mesh, do not show the third peak.

It is clearly visible that unstructured trimmed mesh results (EADS and Boeing) give almost identical results.

\section{Velocity Profiles - FC7}

\section{Overview}

FC7 has conditions $M_{\infty}=0.304, \alpha=11.89^{\circ}, R e=44.4 \times 10^{6}$. These are similar to FC19, and the flow topology is also similar. For this case boundary layer measurements are available.

\section{Velocity Comparisons}

Figure 5 shows the comparison of measured and predicted boundary-layer profiles for rake locations $\# 3, \# 4, \# 7$, and \#5, respectively, for all RANS solutions ${ }^{7-10,12-14,16}$ shown, USAFA $^{15}$ and CFL3D results. ${ }^{1}$ The agreement with flight test data is very good for three of four positions \#3, \#4 and \#5. In position \#7 the spread of all results is rather large. Some of the results show "jet-type" flow (USAFA, NASA unstructured). On the other side is EADS which predicts lower velocities. This rake is located underneath the separation line between primary and secondary vortex and so the flow is complicated in this region. Inspection of the results shows that the structured meshes results have the smallest spread, especially in position \#5 and \#7 compared to other results. The largest spread is in the common unstructured results.

A comparison of steady versus unsteady results is also interesting. Figure 6 shows the envelope of velocity profiles for all RANS solutions in black with unsteady DES, DDES and Hybrid RANS-LES of USAFA and $\mathrm{KTH} / \mathrm{FOI}^{20}$ in two positions where comparisons are the most interesting - position \#5 and \#7. The scatter between RANS data at position \#5 (Figure 6(a)) is large which is also true for different unsteady methodologies. The KTH/FOI DES result shows the largest deviation to the average result whereas USAFA DES lie on the boundary of RANS models interval. USAFA-DDES and KTH/FOI Hybrid modeling moves velocity profiles closer to flight test data and into the cloud of RANS results.

The results at rake \#7 shown in figure 6(b) have large scatter. The KTH/FOI hybrid model shows good agreement with flight data. The DES model predicted velocity profile is on the boundary of the RANS results envelope. USAFA deviates from other results showing 


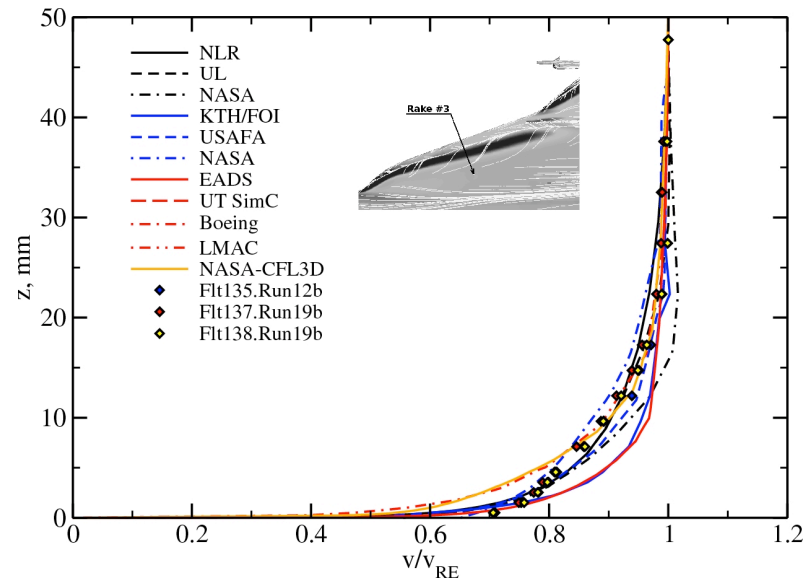

(a) Rake \#3

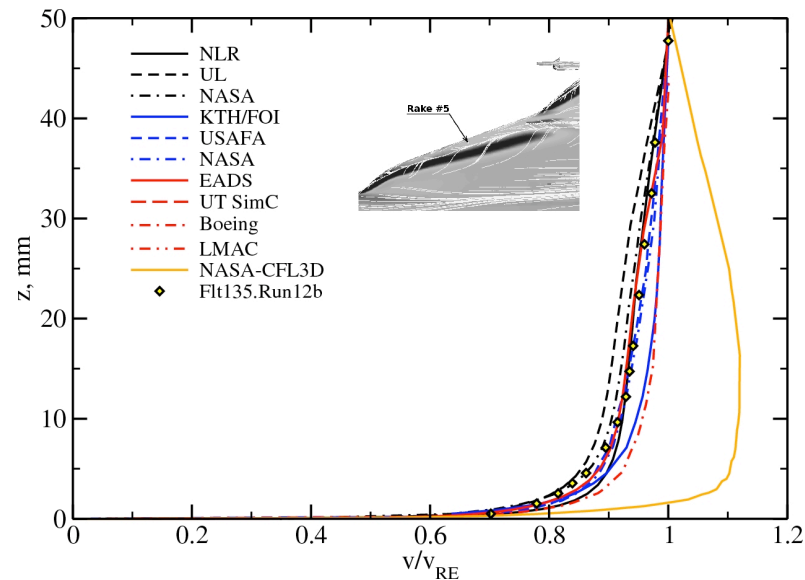

(c) Rake \#5

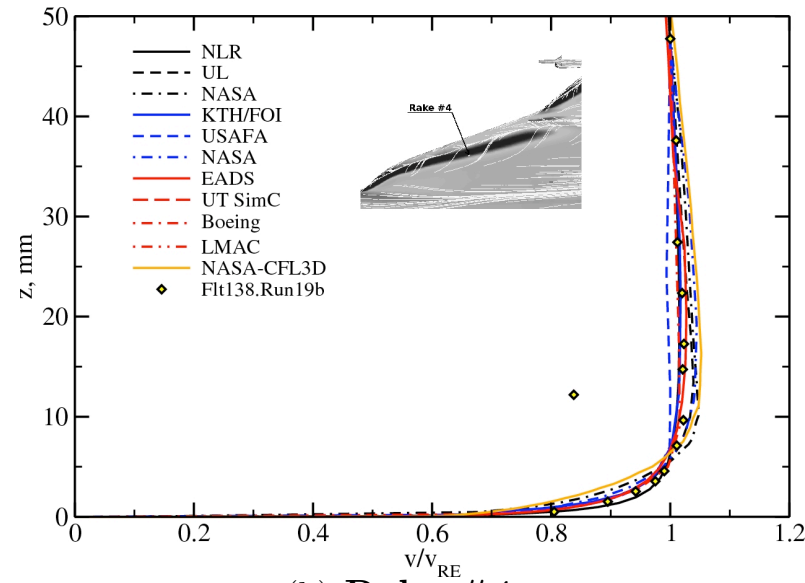

(b) Rake \#4

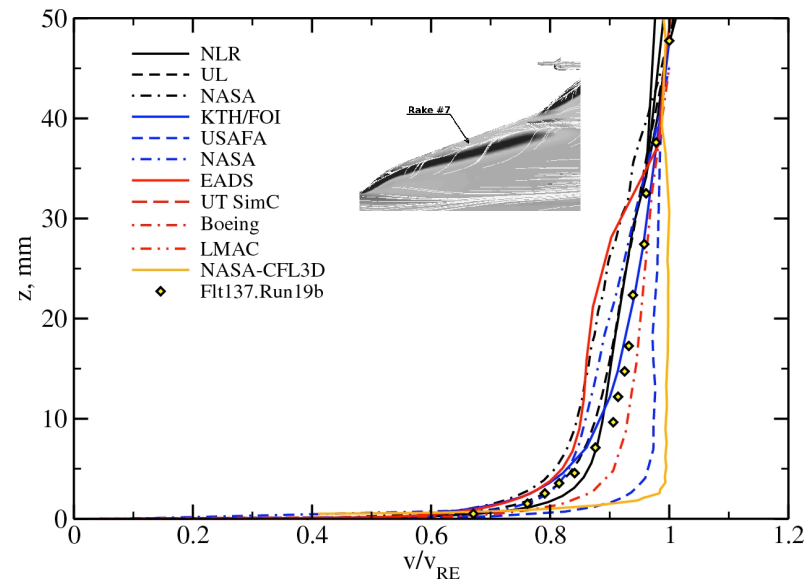

(d) Rake \# 7

Color coding: Black - Common structured mesh

Blue - Common unstructured mesh

Red - Trimmed unstructured mesh

Figure 5. FC07, velocity profiles comparison. Details about grids ${ }^{3}$ and solvers ${ }^{4-16}$ used $^{-1}$ are given in the references, also refer to Table 1 . 


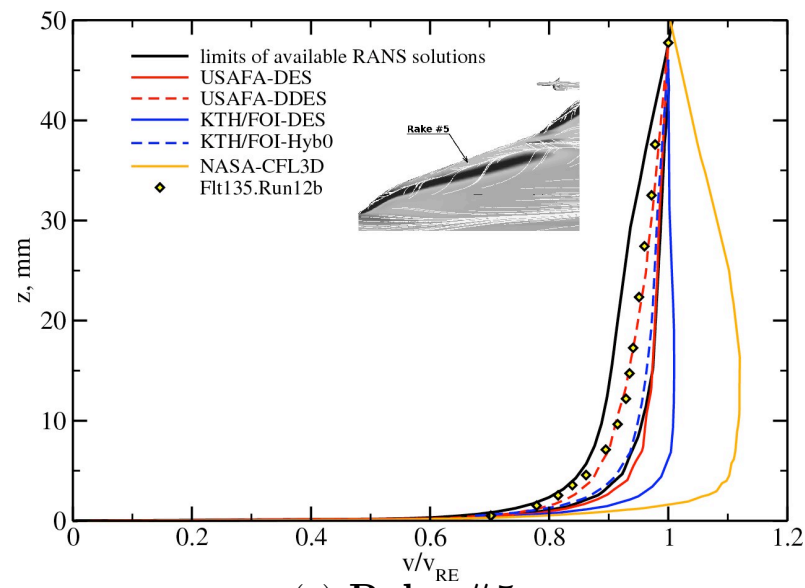

(a) Rake \#5

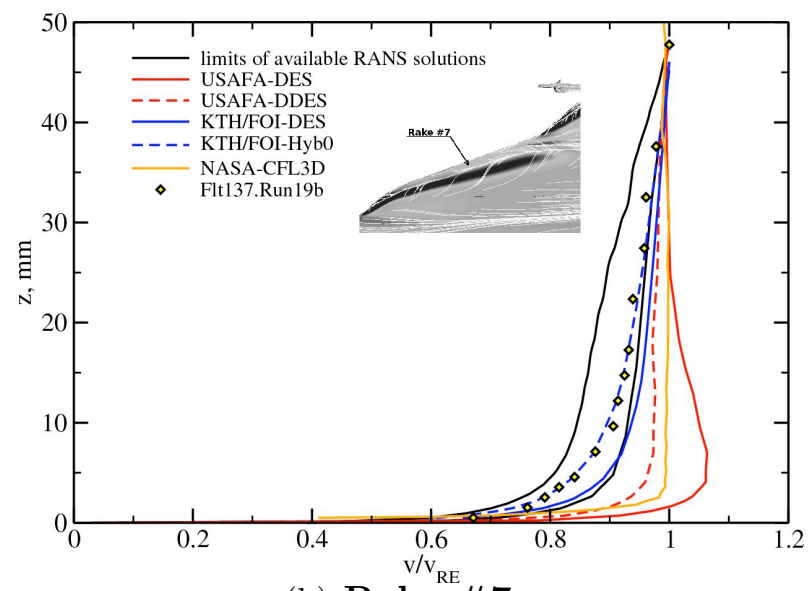

(b) Rake \# 7

Figure 6. FC07, velocity profiles comparison; effect of turbulence modeling and timeaccuracy. Details about grids ${ }^{3}$ and solvers ${ }^{4-16}$ used are given in the references, also refer to Table 1.

substantial improvement of the DDES over DES methodology.

The DES results have trouble in predicting velocity profiles in regions of highly stretched cells. One possible reason for this could be that the DES model does not use RANS in the boundary layer but switches to LES because the grid is so refined in the streamwise direction, resulting in a false log layer. Since the outer edge condition is used in the scaling for the comparisons, this can result in the jet like profile seen in the USAFA results. The DDES model forces the solver to stay RANS in the boundary layer even with refined grids in the boundary layer. These rakes are placed very close to the leading edge with a very refined grid to capture the leading edge suction peaks and therefore are susceptible to this problem. The overall pressure results are fairly insensitive to this but the boundary layer rake data and skin friction DES results seem to be suffering from the problem. The DDES model improves the situation for rake \#5, but less so for rake \#7.

\section{Results at Sideslip Flight Conditions - FC50, FC51}

Two additional flight conditions are side-slip conditions at angle of attack around $\alpha=13^{\circ}$ and subsonic Mach numbers around $M=0.44$ and $R e=39 M$. These two conditions denoted FC50 and FC51 have the value of side-slip angle $\beta=5.31^{\circ}$ (FC50) and $\beta=4.58^{\circ}$ (FC51).

The effect of a sideslip angle is to decrease the effective leading edge sweep angle of the windward-side wing and to increase the leading edge sweep angle of the leeward-side wing. This effective decrease of sweep angle may cause vortex breakdown on the windward side to occur at a lower angle of incidence than it would occur with no sideslip. In addition the reduced sweep angle may cause a double vortex structure. ${ }^{21}$ 
These two flight conditions were not a main focus of the CAWAPI team. Two cases were however computed by some partners. Goertz et $a .^{5}$ report on a study that outlines the effect of turbulence models and unsteadiness of the solution which seems to be significant for BL70 up to BL184.5.

\section{Transonic Flow Case}

\section{A. Overview}

FC70 has conditions $M_{\infty}=0.97, \alpha=4.3^{\circ}, R e=88.8 \times 10^{6}$. This condition has the smallest angle of attack of all the test flights considered, and a high subsonic freestream Mach number. Hence reduced-strength vortical-flow phenomena over the upper surface and strong transonic effects are expected. It must be pointed out that there is some degree of uncertainty in the flight-test data concerning whether, and how much, a flap was deflected during the measurements. Transonic effects are also very sensitive to flight conditions, and even weak interactions can lead to substantial changes in flow structure.

\section{B. Force/Moments Comparison}

Table 4 shows the comparison of force and moments. The statistical analysis of the data

Table 4. Flight condition FC70, force/moment coefficients. Details about grids ${ }^{3}$ and solvers $^{4-16}$ used are given in the references, also refer to Table 1.

\begin{tabular}{lcccccrc}
\hline \hline Coefficient & NLR & KTH/FOI & USAFA & NASA - unstr & EADS $^{\dagger}$ & UT SimC & LM Aero \\
\hline$C_{L}$ & 0.11682 & 0.12276 & 0.12044 & 0.12080 & 0.12641 & 0.12510 & 0.12412 \\
$C_{D}$ & 0.04077 & 0.03901 & 0.04193 & 0.05179 & 0.01959 & 0.04661 & 0.04464 \\
$C_{D_{f}}$ & 0.00674 & 0.00573 & - & 0.01018 & - & 0.00678 & - \\
$C_{M}$ & 0.00219 & -0.00103 & -0.00153 & -0.00129 & -0.00452 & -0.01385 & -0.00289 \\
\hline \hline
\end{tabular}

$\dagger$ Data without inlet and nozzle surface.

from Table 4 is shown in Table 5. Analogously to Table 3, the population mean $\hat{\mu}$ and the value $k \hat{\sigma}$ are useful to analyze the contributed solutions. Again, one of the seven solutions is found to be an outlier. The spread of solutions is for this condition less than for FC25. This is found by comparing the values for $k \hat{\sigma}$ between Table 5 and Table 3 . At FC70 the disparity between the computations is lower than for FC25, i.e. the various computations give very similar results.

\section{Overall Vortical Flow Features}

Figure 7 presents sectional spanwise contours of iso-total-pressure and skin friction lines. The contour plots indicate that the fully developed and coherent vortex structures that 
Table 5. Flight condition FC70, force/moment coefficients - statistical analysis. Details about grids $^{3}$ and solvers ${ }^{4-16}$ used are given in the references, also refer to Table 1.

\begin{tabular}{lcccc}
\hline \hline Coefficient & $\hat{\mu}$ & $k \hat{\sigma}$ & \# identical solutions & \# outliers \\
\hline$C_{L}$ & 0.12276 & 0.00571 & 6 & 1 \\
$C_{D}$ & 0.04193 & 0.01782 & 6 & 1 \\
$C_{D_{f}}$ & 0.00676 & 0.00357 & 4 & 0 \\
$C_{M}$ & -0.00153 & 0.00940 & 6 & 1 \\
\hline \hline
\end{tabular}

have been observed in the previous flight conditions, namely primary and secondary vortices substantially above the inner and outer wings, are not present in this flight condition. The convergence-divergence of skin friction lines do show the confluence of vortical layers near the mid-span of the wing that likely separates and re-attaches inboard near the fuselage. Separation does seem to occur, but the lift-off appears to remain close to the upper surface, either just in, or just above the boundary layer. The flow does separate from the airdam/actuator-pod and a small vortex seems to develop. Notice, however, that no vortex is shed from the outer wing nor is there any sign of a secondary vortex over the inner wing, as found in the other cases.

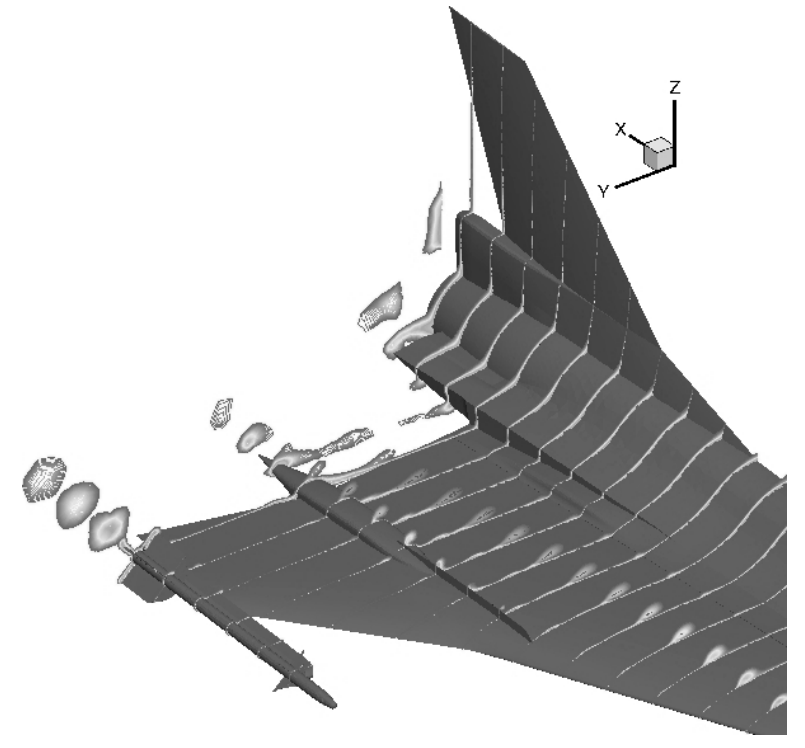

(a) Sectional spanwise iso-total-pressure contours. ${ }^{12}$

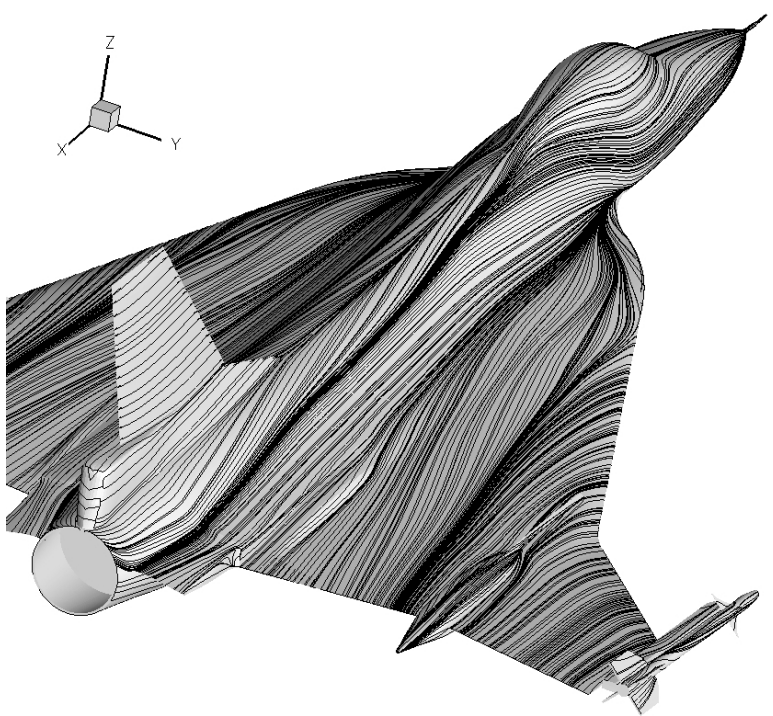

(b) Skin friction lines. ${ }^{7}$

Figure 7. Low-lying vortices over upper surface for FC70. Details about grids ${ }^{3}$ and solvers $^{4-16}$ used are given in the references, also refer to Table 1.

As shown by Fritz et al., ${ }^{6}$ at BL55 there is a shock wave at approximately $5 \%$ chord due to the decelerating effect of the low sweep of the inner-wing fairing. There is another shock wave at about $30 \%$ chord on the upper surface. In the computations there is also a shock at $20 \%$ chord of the lower wing surface. There are, however, no measured values 
at this location to confirm it. Along BL70 and BL80 several computations agree with each other, but not with the measurements. The computed results show a shock on the upper surface located well upstream of the shock shown by flight data. The last two butt-lines, BL153.5 and BL184.5, are on the outer wing where the flow is influenced by the airdam, crank and missile. All computational results over-predict the suction peak near the leading edge; this could be due to the leading-edge flap on the outer wing that was reported to be deflected upward (from negative $5^{\circ}$ to negative $9^{\circ}$ ) while the trailing-edge flap was deflected $2^{\circ}$ downward in the flight test. Differences begin to grow in the computed results especially in the aft part of the chord. At BL153.5 the measurements might suggest a shock just

before $80 \%$ chord, and just before $60 \%$ chord in BL184.5. This is in disagreement with the computations which could be due to the in-flight deflected trailing-edge flap, which is not modelled in the computations.

A general inspection of the butt-line $C_{p}$ comparisons of Fritz et al. ${ }^{6}$ prompts the overall comment that the computed data agree very well among themselves but differ substantially from the measurements, except at BL55 and BL95 where all results are in fairly good agreement. If we accept the explanation of the negatively-deflected leading-edge flap to be the cause of the discrepancies at BL153 and BL184.5, then it is the discrepancies at BL70 and BL80 that beg for explanation. The work of CAWAPI members therefore focused on carrying out several additional activities to explain the cause of this discrepancy.

\section{Investigating Possible Causes of Discrepancies}

The investigations are divided into two categories: one, targeting possible computational reasons, and the other, targeting possible reasons which may have appeared during flight test.

\section{Activities to Eliminate Possible Numerical Effects}

The activities targeting possible numerical causes were: Mesh dependency study, turbulence model effect study, physical model study, effect of unsteadiness and effect of different formulation of boundary conditions.

- Mesh dependency study by mesh adaptation. EADS ${ }^{6,12}$ carried out a study investigating mesh dependency, particularly in and above the boundary layer. Manuallyadapted and solution-adapted grids were used. The finest grid solutions, both manually and solution-adapted, show a weak primary vortex over the inner part of the wing, whereas the coarser-mesh solutions show this vortex only in the most forward part of the wing. The vortical features obtained on the adapted grid are shown in 
Fig. 7 However, the presence of this vortex does not change the overall character of the solution, ${ }^{6}$ which may indicate that the mesh adaptation has not progressed far enough.

- Reynolds-number effect - mesh $y^{+}$. The grids which were used for all other FC calculations had the value of $y^{+}$set by the requirement of subsonic flight where the Reynolds number is about half of the value of FC70. A mesh made at EADS which corrected this anomaly showed no difference in result.

- Turbulence modeling effects. $\mathrm{KTH} / \mathrm{FOI}^{11}$ and $\mathrm{NASA}^{14}$ carried out an investigation aimed at testing different turbulence models and their possible effect on results. No major difference was observed.

- Unsteady calculations. USAFA $^{15}$ carried out unsteady calculations using SARCDES. Their solution is very similar to the RANS solutions.

- Effect of formulation of boundary conditions in inlet and nozzle. $\mathrm{KTH} / \mathrm{FOI}^{22}$ carried out different tests with given values of static pressure at the inlet and total states in the nozzle as well as with mass-flow boundary conditions in the nozzle. No effect from the different boundary-condition formulations was observed.

\section{Activities to Eliminate Possible Flight Test Reasons}

The activities targeting possible flight test reasons for the discrepancies were: effect of incidence and side-slip angle, effect of change in aircraft geometry during flight test, effect of bending of the wing and a check on possible corruption of flight-test data.

- Incidence and side-slip angle effect. EADS and NLR carried out a study with a small value of angle of incidence and side-slip angle. Despite changes in the solutions, the overall characteristics of the results do not indicate that any small change of angle of attack or angle of side-slip would explain the discrepancy between CFD and flight data.

- Effect of change in aircraft geometry. As pointed out in NASA/TP ${ }^{1}$ post flight test analysis, the leading-edge flap was deployed $-9^{\circ}$ during the test. UT SimCenter carried out tests with a deformed mesh to investigate the possible effect of the flap deflection. The result of this analysis shows that the airdam blocked any influence of the outboard flowfield on the inboard pressure field and thus the flap deflection does not explain the discrepancies between computational results and flight test.

- Effect of wing bending. The structures engineers designed the standard F-16 aircraft to sustain $9 \mathrm{~g}$ maneuvers which means that the wing is stiff. At high loading it 
would be significant, but at a cruise condition the bending should be small. The computations agree well with the flight-test data at the first inboard span station (BL55) and the mid-span station (BL95). If bending occurred, it would certainly cause discrepancies also in these two stations. Furthermore, the bending effect would have to be visible at the high incidence angle flight conditions, which was not observed.

- Check on flight-test data. There is no indication in the flight-test reports that the flight data had been taken incorrectly or that the data has been corrupted. As a further check, KTH/FOI calculated the flowfield at FC68 and FC69 which are near transonic conditions. FC68 seems to be mostly subsonic and the results are in good agreement with flight data. At FC69, where the supersonic portion of the flow is much larger than that for FC68, but smaller than that for FC70, the differences between computations and flight data begin to grow substantially, but they are still less than at FC70.

The last item suggests a possible cause and effect. The conditions for FC68 are $M_{\infty}=0.90$, $\alpha=3.7^{\circ}$; and for FC69 they are $M_{\infty}=0.95, \alpha=3.6^{\circ}$. The only value that changes significantly between the three FC is the Mach number, from 0.90 to 0.97 . When there is little supersonic flow (hence no shocks) the agreement is good, and when there is most supersonic flow and strongest shocks, the agreement is worst. We hypothesize that the accurate simulation of a shock wave-vortex interaction phenomena could be required to resolve the discrepancies.

\section{E. Highly-Adapted Meshes to Resolve Interaction of Vortex with Shock}

The KTH team undertook an extensive study of the FC70 flowfield by generating highlyadapted meshes, as shown in Table E. KTH began with the surface triangulation of the common unstructured mesh, and after some cleaning, arrived at 158 thousand surface triangles. Substantially more prism cells were added (6.12 million) in the boundary layer and fewer tetrahedral cells were included outside than in the common mesh. This became the KTH initial RANS grid. Running on this grid, the Edge code automatically adapted the mesh using a gradient sensor to produce the KTH singly-adapted grid with over 11 million cells in total. This is more than three times the number in the common grid. A second level of adaptation is not possible because the resulting mesh would go beyond what the KTH computing facilities can handle. However using the Euler solver, additional levels of adaptation could be handled because of the lighter computational load. Starting from the surface triangulation of the common grid, KTH created the initial Euler grid of 8.99 million nodes, and this grid was adapted three times. The first adaptation used the gradient sensor 
to mainly seek out shock waves, the second adaptation used the $\lambda_{2}$ sensor ${ }^{23}$ that tracks vortex cores, and the third adaptation was again the gradient sensor. The result is the KTH triply-adapted grid of 902 thousand surface triangles and 16.2 million nodes.

Table 6. Computational grid sizes (pyramidal elements are included in the total volume cells counts).

\begin{tabular}{llcccc}
\hline \hline Grid & & $\begin{array}{c}\text { Surface } \\
\text { triangles } \times 10^{3}\end{array}$ & $\begin{array}{c}\text { Prism } \\
\text { cells } \times 10^{6}\end{array}$ & $\begin{array}{c}\text { Tetra } \\
\text { cells } \times 10^{6}\end{array}$ & $\begin{array}{c}\text { Total volume } \\
\text { cells } \times 10^{6} / \text { nodes } \times 10^{6}\end{array}$ \\
\hline \multirow{2}{*}{ RANS } & common & 160 & 1.44 & 10.49 & $11.94 / 2.54$ \\
& KTH initial & 158 & 6.12 & 7.40 & $13.56 / 4.36$ \\
& KTH singly & 433 & 16.81 & 14.65 & $31.52 / 11.03$ \\
& adapt. & & & & \\
Euler & KTH initial & 158 & - & 8.99 & $8.99 / 1.53$ \\
& KTH triply & 902 & - & 94.71 & $94.71 / 16.20$ \\
& adapt. & & & \\
\hline \hline
\end{tabular}

The wing of the F-16XL is swept and sharp enough that a vortex shed from the leading edge is expected even in an Euler simulation, although the location of where it is shed may not be correct in reality. And that is precisely what the Euler solution obtained on the triply-adapted mesh indicates. Figure 8 presents the surface meshes on the upper wing surface. The grid in the top-half of the figure is the Euler mesh adapted 3 -fold yielding a total of 902 thousand triangles on the total surface (upper and lower), and the bottom half shows the unstructured common mesh with 160 thousand triangles on the total surface. The inset box is a zoom of the region where the leading-edge vortex intersects the shock wave. The adaptation procedure picks out the major flow features and these are clearly visible in the intensity of the triply-adapted grid clustering in the Euler mesh, compared with the rather uniform grid spacing in the common mesh. The dark, span-wise band is the result of two gradient-based adaptations to the shock wave. Of the three dark horizontal bands, the longest and middle one is the footprint of the primary vortex core as it shows three levels of adaptation, two gradient based ones, and decisively, one vortex-tracking $\lambda_{2}$-based adaptation. This dark band results from the vortex interacting with the shock wave, and persisting downstream of it. The shorter bands above and below this result from just the gradient-based adaptation.

Figure 9 presents the Euler solution computed on the triply-adapted grid. Streamlines superimposed over iso-Mach contours on the upper surface of the inboard wing are shown in the top half, and isobars of $C_{p}$ are shown in the bottom half. The impinging of supersonic streamlines in Figure 9 demonstrates that a vortex is shed from the fore section of the wing leading edge upstream of the shock wave. The diverging supersonic streamlines identify 


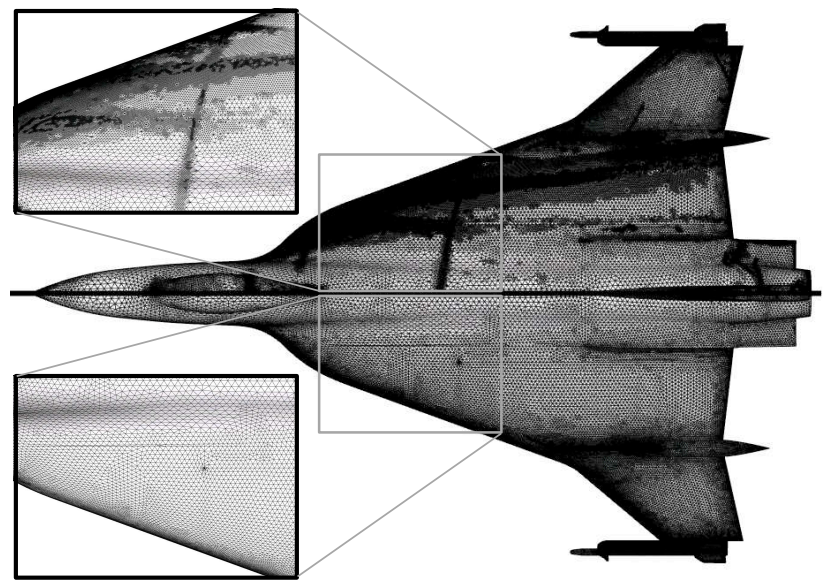

Figure 8. Surface grids on upper surface; top half: triply-adapted mesh for Euler, lower half: common RANS mesh.

the location where the flow re-attaches after the vortex lift-off. The supersonic streamlines inboard of the re-attachment line decelerate to subsonic speed across the shock wave (blue region). The streamlines under the vortex however do not decelerate to subsonic speed but persist through the shock wave. Outboard of the primary separation line the supersonic streamlines again decelerate to subsonic speed across the shock. That a supersonic jet of vortical air issues through the shock, in effect annihilating the re-compression to subsonic flow, the usual shock-wave effect, is an important finding.

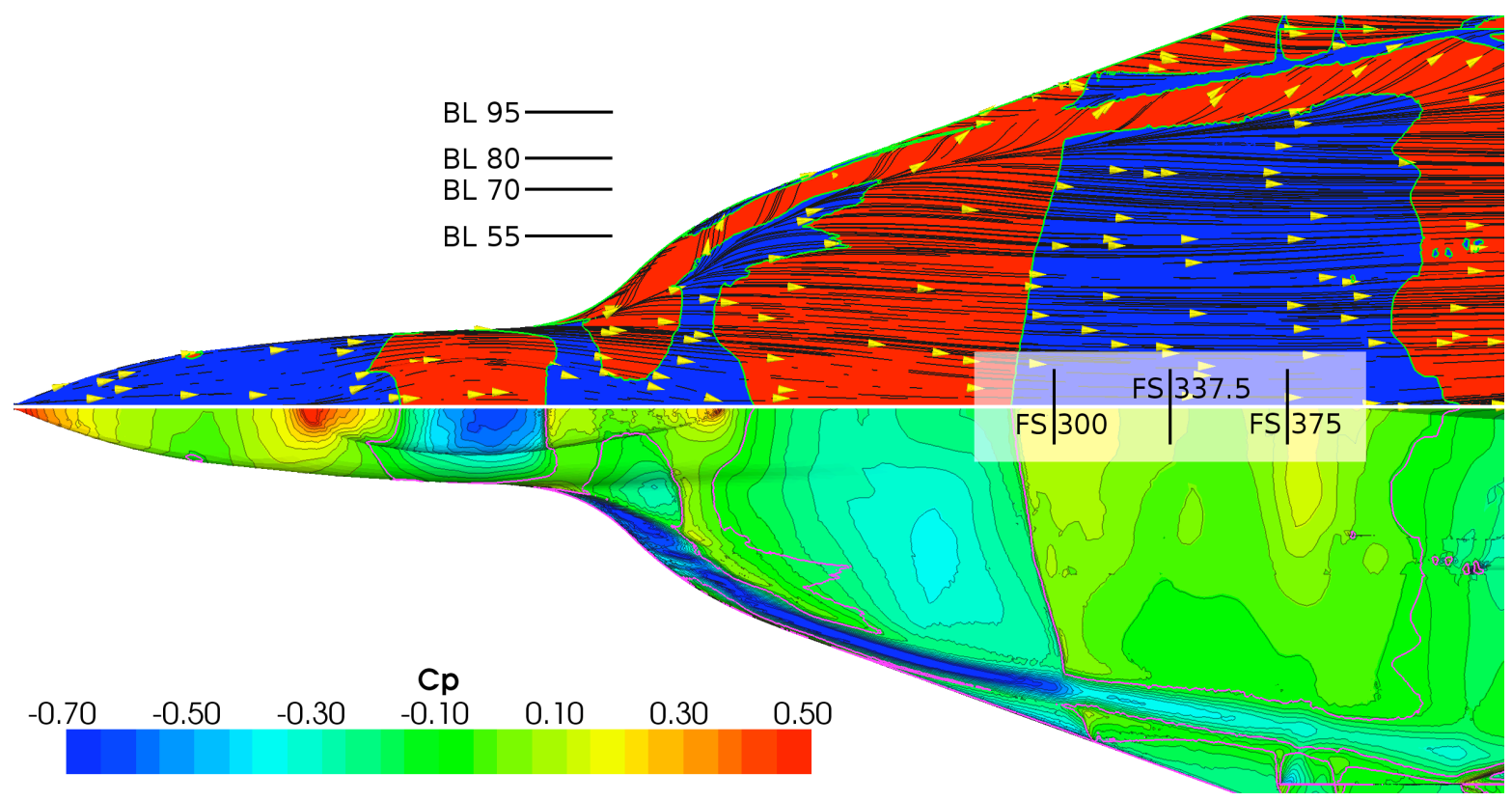

Figure 9. KTH inviscid solution on upper surface with triply-adapted mesh; top half, streamlines superimposed over surface Mach number (Blue: $M<1$; green: $M=1$; red: $M>1)$; bottom half, isobars of $C_{p}\left(C_{p}^{*}=-0.052\right.$ magenta). 
Figure 9 reveals further features of this shock wave-vortex interaction. Ahead of the shock wave the convergence of the surface streamlines that form the lift-off of the shear layer that feeds the vortex is almost entirely supersonic flow, as is the re-attachment of these streamlines. In the vortical jet downstream of the shock wave, this lift off and subsequent re-attachment occur in substantial regions of subsonic flow, i.e. the supersonic streamlines re-attach by decelerating to subsonic flow presumably through a shock wave. These features have not been seen in any of the previous RANS solutions, presumably because of insufficient grid resolution of both the shock and the vortex.

A RANS solution is needed to corroborate what was found in the Euler solution. Unfortunately the RANS solution computed on the singly-adapted KTH grid shows only the suggestion of the vortex shed from the leading edge, somewhat inboard of that shed in the Euler solution, interacts with the shock wave, but has insufficient strength to penetrate through it. Therefore we can only speculate that with still higher grid resolution, the RANS solution would proceed in the direction of the Euler solution.

\section{F. Comparisons along BL Sections}

Fritz et al. ${ }^{6}$ found the largest $C_{p}$ discrepancies to be at BL70 and BL80, and thus this region in the Euler and RANS solutions are of prime interest to investigate. Figure 10 presents a chordwise cut through three different unstructured grids in the BL80 section to illustrate the effects of mesh adaptation. The inset frame is a magnification of the first third of the wing chord that contains the region of the shock-vortex interaction. The dark bands of clustered cells in Figs. 10(a) and 10(b) visualize the location of the shock in section BL80 and the density of cells over the upper surface, features not seen in Fig. 10(c) of the common grid.

Figure 11 compares the values of $C_{p}$ computed on the triply-adapted Euler grid, the singly-adapted RANS grid and the common RANS grid with the values measured in the flight test for FC70. In BL55 all three computed values agree reasonably well with each other and with the flight-test results, although at the first $10 \%$ of the chord the computed results over predict the suction there. In the BL95 section the two RANS results agree with each other and with the measured data. The Euler results, however, differ from these. The flow does not compress through the shock to the subsonic values at about $15 \%$ chord as it does for the RANS result and the measurements. Instead it expands further out to about $20 \%$ chord and then gradually compresses (isentropically) to about $40 \%$ chord, i.e. there is no evidence that a shock is transversed. The following explanation is offered for this. If BL95 were to be marked in Fig. 9 it would pass through the region of interaction between the vortex and the shock wave. The footprint of the vortex is supersonic, hence no compression, which only comes further downstream where section BL95 passes outside of the vortex footprint. We can also conclude then that the position of the vortex in the Euler solution is not realistic. 


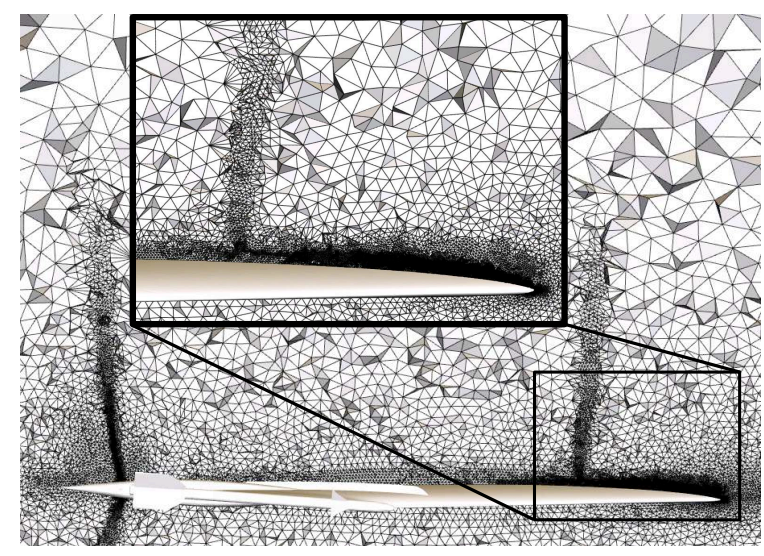

(a) KTH Euler mesh, triply adapted.

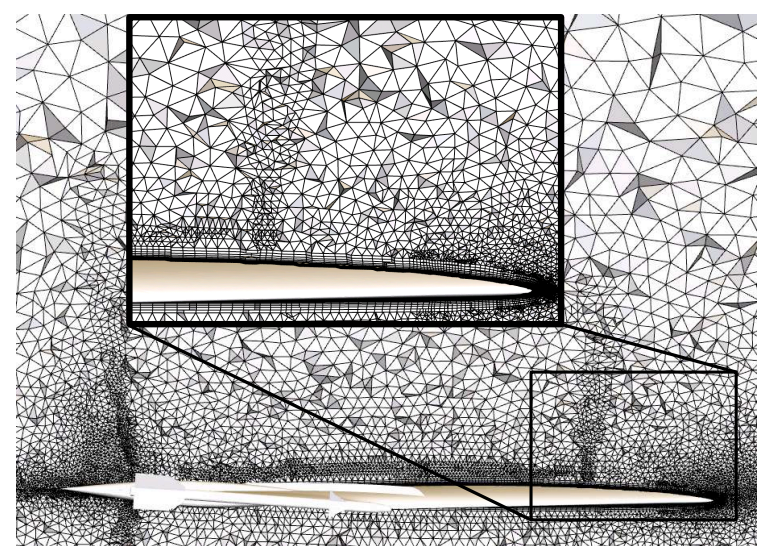

(b) KTH RANS mesh, singly adapted.

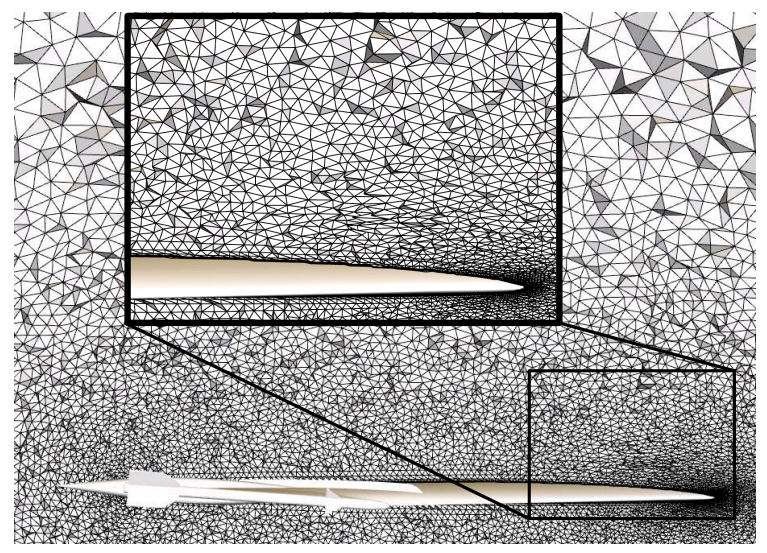

(c) Common RANS mesh, no adaptation.

Figure 10. Chordwise cut through three unstructured grids in BL80 section. 


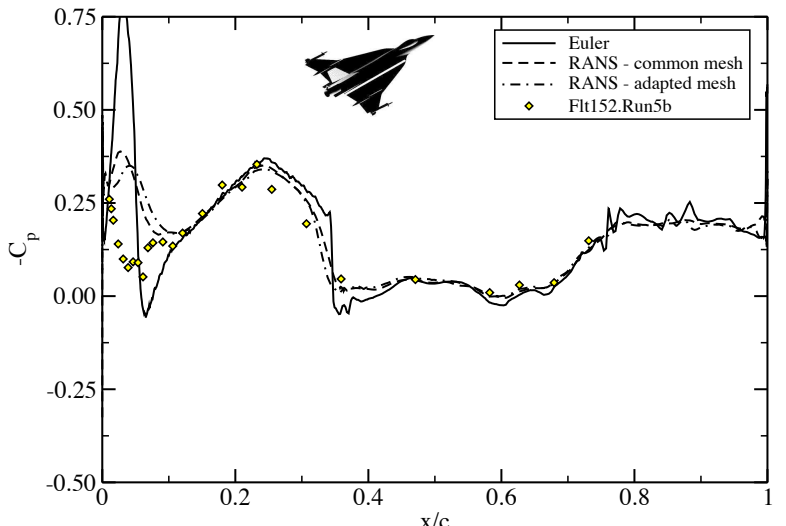

(a) BL55

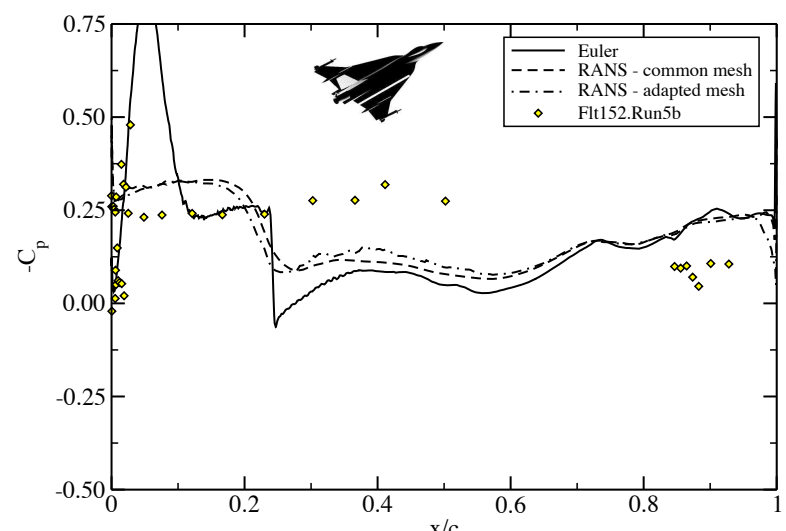

(c) $\mathbf{B L 8 0}$

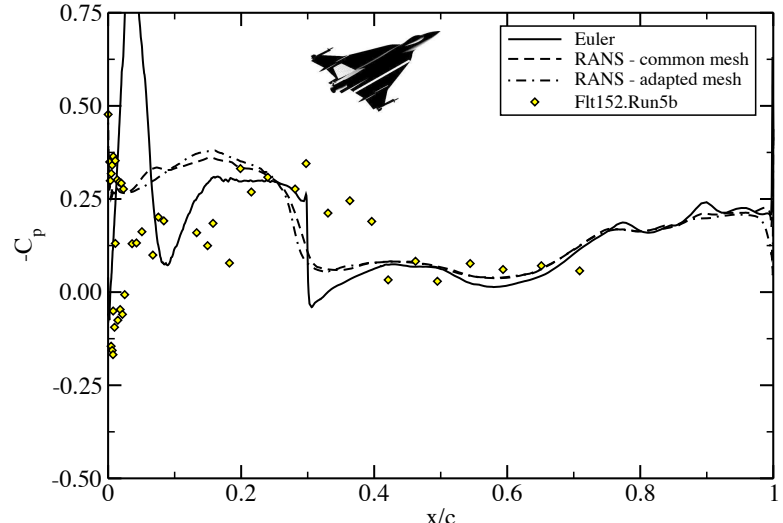

(b) $\mathbf{B L} \mathbf{x}^{\mathrm{x} / \mathrm{c}} \mathbf{0}$

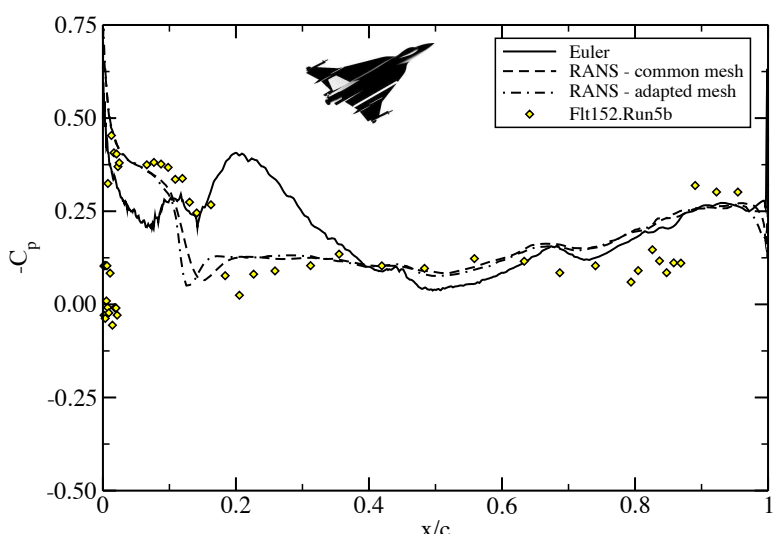

(d) BL95

Figure 11. Chordwise comparison of computed and measured surface $C_{p}$ for case FC70. 
Again at BL80 the two RANS result agree with each other and indicates the shock just past the $20 \%$ chord location. The Euler result displays a substantial suction peak at the nose and then a very sharp shock just past $20 \%$ chord that confirms the RANS computation, but all three results are in contradiction to the measurements which indicate supercritical flow to at least 50\% and maybe further. What is found at BL95 and BL80 then suggests the following explanation. If we could compute RANS with sufficient resolution, the vortex footprint would remain supersonic through the shock, as we see in the Euler solution, but it would lie inboard of the location of the Euler result. We see in BL95 how the $C_{p}$ values remained supercritical across the shock wave, but in RANS this would occur at approximately BL80. If this suggestion holds true, then the flight tests would confirm the passing of the supercritical vortex through the shock wave. Lastly in BL70 the two RANS values agree closely, and the sharp shock in the Euler results is in reasonable agreement with RANS, but the measurements show no evidence of the shock. The measurements rather show a continuous rise and fall in $C_{p}$ reminiscent of the Euler computed values at BL95, in other words the BL70-line could also be cutting across the vortex footprint near the shock-vortex interaction zone.

\section{G. Comparisons along FS}

Let us further investigate the nature of the shock-vortex interaction and especially the character of the flow downstream of the shock wave. For this purpose it is useful to look in spanwise sections at constant chord position. Figure 12 shows the span-wise cut through the three different unstructured grids in the FS337.5 section to illustrate the effects of mesh adaptation on the vortex core just behind the shock wave. The upper inset frames of Figures 12 (a) and 12(b) present a magnification of the outer half of the cross-section. The lower inset frames present the same area as for the upper magnification inset, but here the totalpressure-loss iso-lines are plotted for the respective case. The left-half of Fig. 12(a) presents the Euler mesh with three levels of adaptation, and the right half presents the common unstructured mesh for RANS with no adaptation. The left-half of Fig. 12(b) presents the RANS mesh with one level of adaptation, and the right half presents the common unstructured mesh for RANS with no adaptation.

The large dark region of clustered cells in the center of the left inset frame of Fig. 12(a) is the grid adaptation to the vortex core and gradients in the Euler solution and indicates the relative location of the vortex core along the span and above the wing. In comparison to the left inset it also indicates the increased grid resolution relative to the common grid. Compare this with the less dark region of clustered cells along the right edge of the left inset frame of Fig. 12(b) which is the corresponding grid adaptation to the vortex core in the singly-adapted RANS solution, less than that for triply-adapted Euler grid but more than 
that for the common grid. The total-pressure-loss iso-lines reveal the same picture as the analysis of the adapted mesh regions. The compact, circular iso-lines of the Euler solution in Fig. 12(a) reveal the location of the compact vortex. The superior resolution of the vortex in the Euler case is clear when comparing the iso-lines of total-pressure-loss to the common solution. By analyzing Fig. 12(b) it is possible to discern the effect of mesh adaptation on the resolution of the vortex for the RANS cases. The location of the vortex of the RANS cases does not change substantially, but the adapted RANS solution shows an improved vortex resolution with a discernible vortex core. This clearly indicates that the vortex core in the Euler solution is about the same distance above the wing but about 15\%-span further outboard than the vortex core in the singly-adapted RANS solution. This finding supports our argument in the previous section that claimed the features seen in the Euler-computed $C_{p}$ values in BL95 are comparable to the flight test values in BL80.

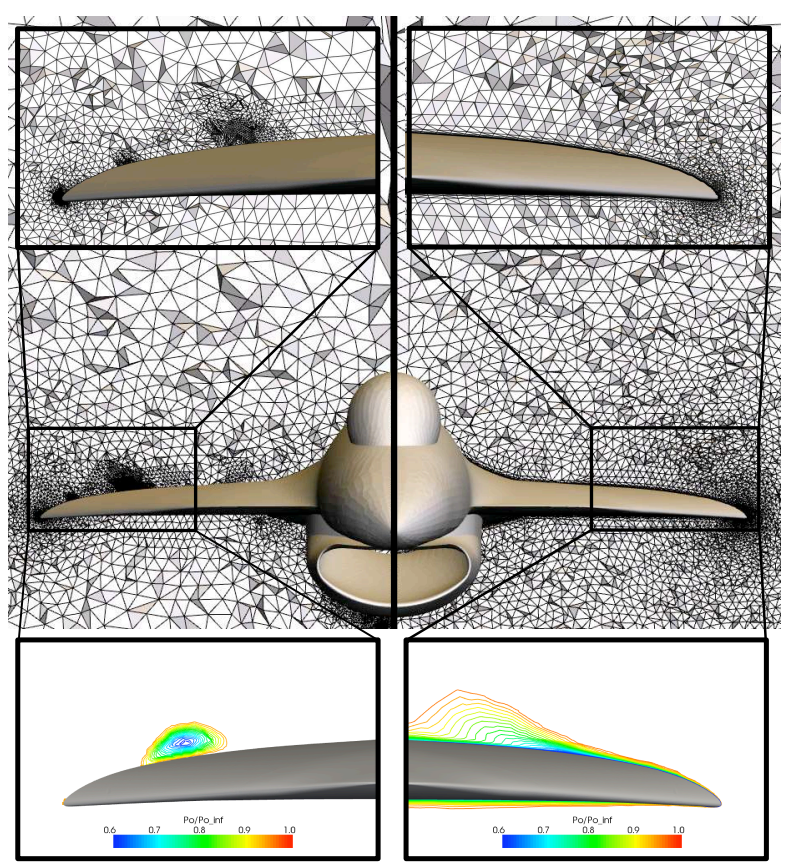

(a) Left: triply-adapted Euler grid; right: common RANS mesh.

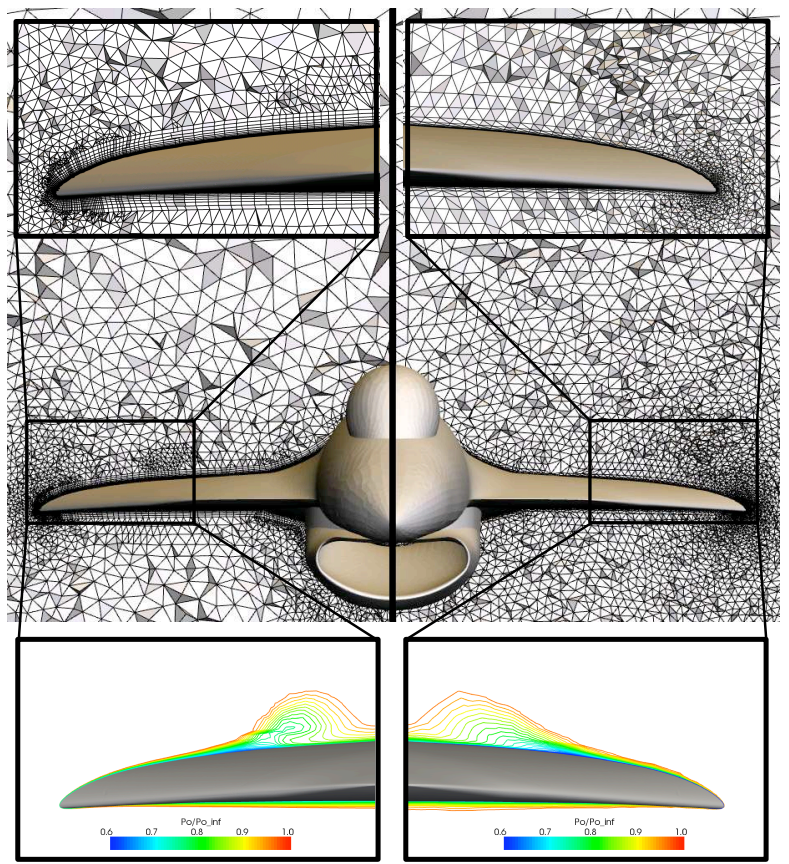

(b) Left: singly-adapted RANS grid; right: common RANS mesh.

Figure 12. Span-wise cuts at FS337.5 for three different unstructured solutions; magnified mesh and total-pressure-loss iso-lines.

In an exactly analogous manner Fig. 13 shows the span-wise cut through the three different unstructured grids in the FS492.5 section to illustrate the effects of mesh adaptation to the vortex core just before the trailing edge of the wing. Now there are two dark regions of clustered cells in zoom frame of the Euler grid (left side of Fig. 13(a)). The inboard adaptation is a result of the very weak leading-edge vortex and the outboard region is due to the vortex shed from the airdam. Although the cell density is lower due to the lesser degree 
of adaptation, two regions in the zoom frame of the RANS grid (left side of Fig. 13(b)) have the identical interpretation, which in fact is consistent with the visualization of the two vortices at the trailing edge in Fig. 7(a). The total-pressure-loss iso-lines at the FS492.5 section reveal the location of either one or two vortices. The location of the air-dam induced vortex is fixed for all three cases. In the RANS solutions, this vortex is located above the actuator pod, whereas the main wing leading edge vortex is recognizable further inboard. For the RANS solutions, the further inboard located leading-edge vortex is so weak at this station that the total-pressure-loss iso-lines show no clear evidence of its location. In the Euler solution the further outboard located leading edge vortex merges with the air-dam vortex. This results in a markedly stronger and wider vortex above the actuator pod.
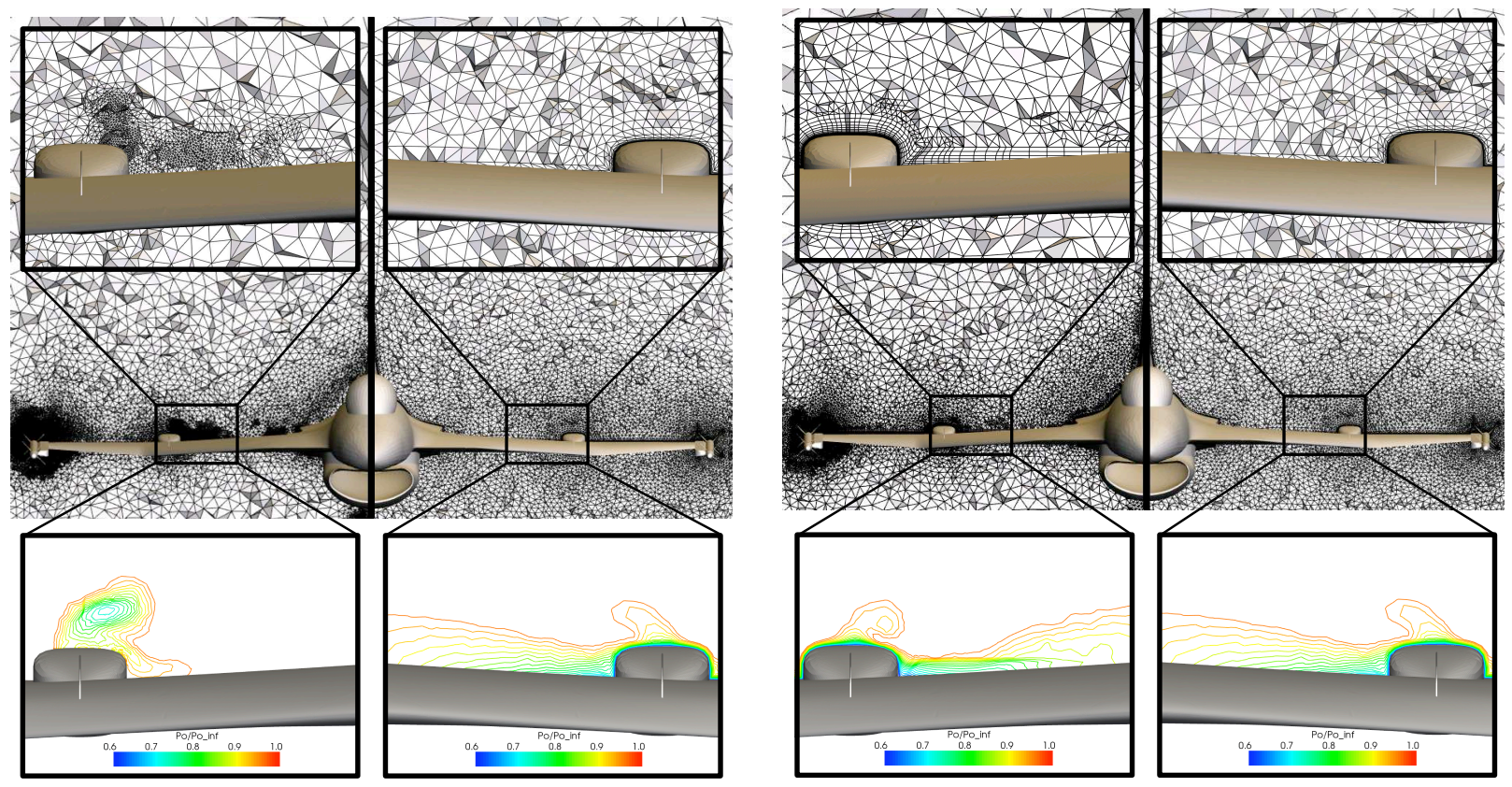

(a) Left: triply-adapted Euler grid; right:(b) Left: singly-adapted RANS grid; right: common RANS mesh. common RANS mesh.

Figure 13. Span-wise cuts at FS492.5 for three different unstructured solutions; magnified mesh and total-pressure-loss iso-lines.

Figure 14 compares the values of $C_{p}$ computed on the triply-adapted Euler grid, the singly-adapted RANS grid and the common RANS grid with the values measured in the flight test for FC70 in the sections FS300, FS337.5, FS375 and FS492.5. In FS300, which is just in front of the shock, the Euler computed values show a suction peak at approximately $85 \%$ span. If we would place this at about $70 \%$ span to account for the vortex in the Euler solution being $15 \%$ further outboard, then this peak would be in fair agreement with the suction peak in the flight test data at about $70 \%$ span. Notice also that there is a small peak in the singly-adapted RANS-grid solution at about this same position, which with further 
adaptation might increase to the level of the measurements. Station FS337.5 is downstream of the shock wave, and the three measured data values clearly indicate a supercritical suction peak. The Euler solution presents a peak, again about $15 \%$ further outboard, and the singlyadapted RANS result shows the emergence of a small peak in the position that agrees with the measured one. Station 375 is at the beginning of the airdam, and the flight data seems to suggest two suction peaks. The inboard one at about $60 \%$ span is the leading-edge vortex suction consistent with the smaller singly-adapted RANS one and the outboard peak in the Euler result. The second peak in the data measured at $80 \%$ span is due to the vortex shed from the airdam, consistent with the two vortices seen in Fig. 7(a). Lastly, the flight-test data in section FS492.5 is too sparse to distinguish the two vortices but the few points that exist are consistent with such an interpretation.

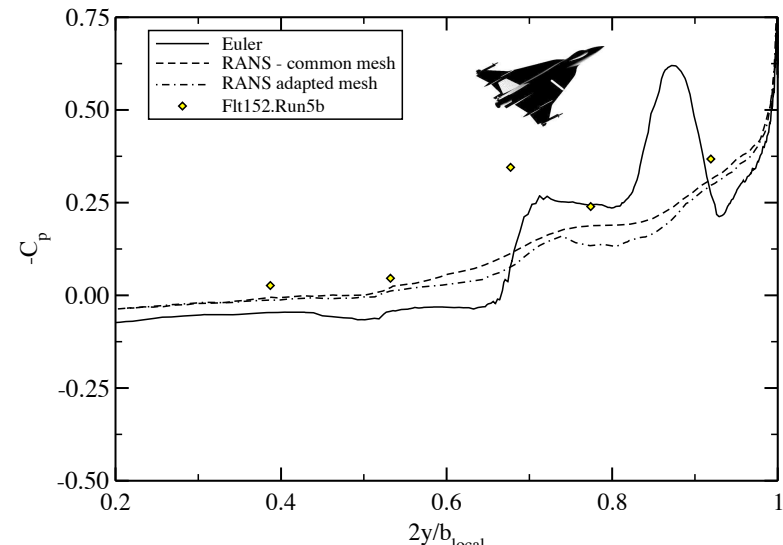

(a) FS300

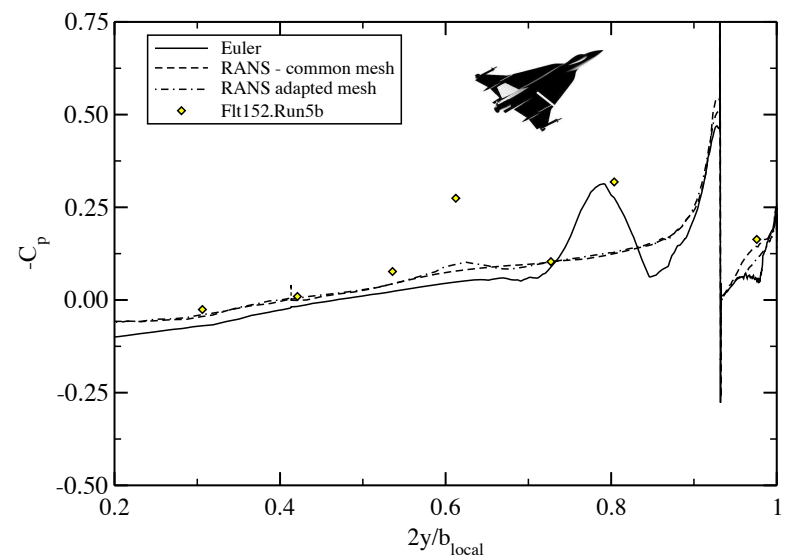

(c) FS375

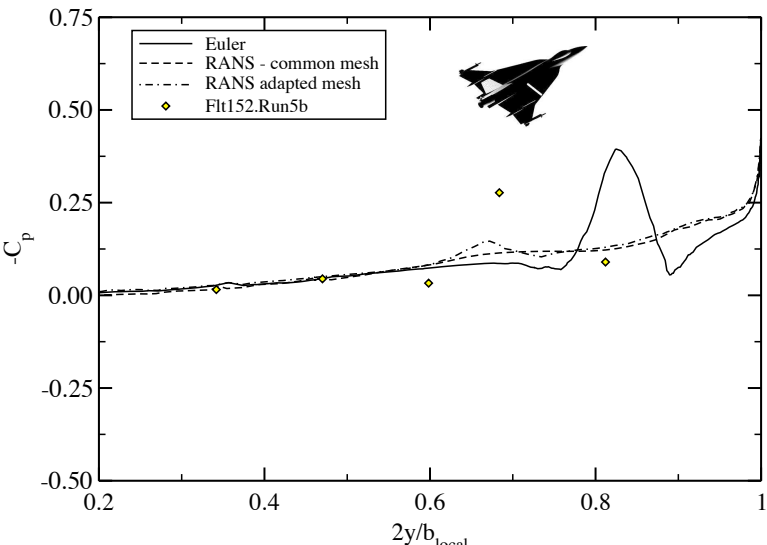

(b) FS337.5

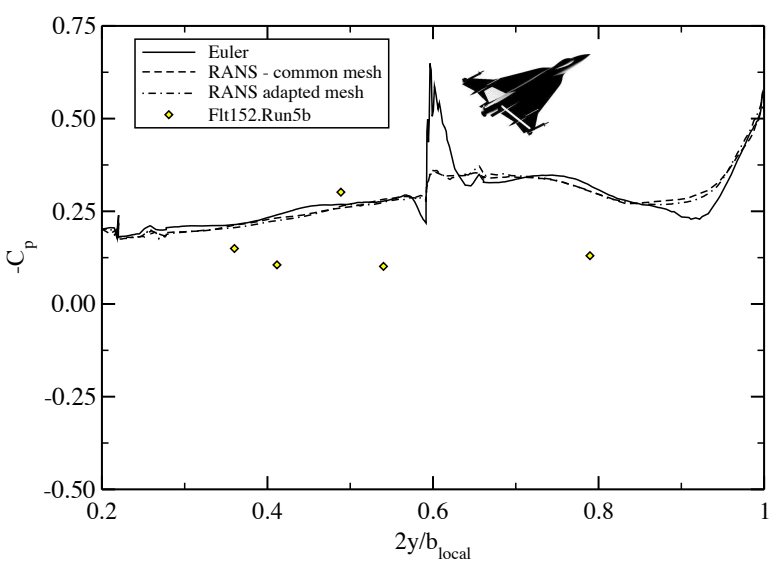

(d) FS492.5

Figure 14. Spanwise comparison of computed and measured upper surface $C_{p}$ for case FC70. 


\section{H. FC70 Concluding Remarks \& Lessons Learned}

Flight condition 70 is the highest Mach-number case examined by all participating organizations, also the highest Reynolds-number case and the lowest angle-of-attack case, so in a sense an extreme of the examined flow conditions. It is the only case examined with interacting supercritical vortex flow and shock waves. Before the CAWAPI team started work on FC70, the major focus was to determine the best practices needed to simulate accurately the vortical flow features and pressure distribution over the aircraft. The geometry used and the mesh generated was the same for all cases including the transonic one. Use of all the grids that lead to reasonable results for all the other test flights did not produce satisfactory results for this flight condition. It took a detailed Euler simulation with a highly adapted mesh to reveal the occurrence of a shock-vortex interaction that significantly changed the surface pressure distribution. One conclusion is if the mesh used offers sufficient resolution to resolve the shock-vortex interaction problem, one then observes a jet of supersonic flow blowing through the shock wave, delaying re-compression to further downstream that seems to be continuous, i.e. without a shock wave. If the goal is to resolve the detailed pressure distribution over the aircraft, then this feature must be resolved.

The lessons learned are:

- At high Mach number, high Reynolds number and low $\alpha$ expect shockvortex interaction This is difficult to resolve because the vortex does not lift very far off the surface and out above the boundary layer.

- $y^{+}$on the surface is not a conclusive indicator that one has a sufficient mesh The mesh must resolve the vortex lift-off and interaction across the entire boundary layer and beyond.

- Adapt mesh for both shock and vortex The occurrence of the supercritical vortex core that persists across the shock wave requires resolving both the shock and the vortex. Adapting to just one of these will miss this combined effect. For example, the grids sufficient for all the other flight conditions, i.e. those that had just vortex phenomena missed the shock-vortex interaction in FC70. It could take well more than an order of magnitude increase in grid cells to resolve the associated interaction region and boundary layer.

- Shock-vortex interaction may be unsteady This phenomena presumably has some unsteady components to it, but this aspect was not investigated here. We expect that these aspects would occur on a scale that would require high grid resolution of the interaction region to be captured. 
There is no reason to expect that the difficulties (and features) encountered with FC70 are in any way specific to the F-16XL airframe. We are convinced that the same type of shockvortex interaction will occur on other similar types of military aircraft at similar (routine) flight conditions.

\section{Perspectives}

\section{A. Progress Since NASA-TP}

In Lamar et al. ${ }^{1}$ flight and wind tunnel test data are presented and are compared with CFD predictions. The code CFL3D was used, which was developed at NASA Langley and is widely used in the United States. A patched multiblock grid was generated on a simplified half configuration of the F-16XL, with the intention to focus grid points on the wing to resolve vortices and shocks. The wing tip missile and launcher, and the vertical fin were removed, although the air dam was retained. The final grid had 1.37 million cells, with the first cell spacing set for the wind tunnel Reynolds number. The flight Reynolds number was computed using a wall function. The turbulence model used was Baldwin-Lomax with the Degani-Schiff modification for vortical flow. The calculations were run on the Cray C-90 and each steady calculation required around 24 hours. The convergence was stopped after the residual had been driven down 2-3 orders, which is unlikely to be sufficient. Finally, the commercial package Fieldview was used to visualize the vortices.

For the vortical flight conditions considered in CAWAPI the $C_{p}$ distributions generally show better agreement inboard. However, suction peaks are under-predicted. One contribution to this is the coarseness of the grid. There were also significant discrepancies between the skin friction and boundary layer predictions and measurements. For FC70 (transonic case), good agreement was again obtained inboard, with very poor comparison with flight measurements outboard of the crank. A possible cause of this was suggested to be the uncertain deployment of the leading edge flaps and the ailerons. It was also stated that the convergence of the transonic case was more difficult.

Computing Power The computing power available to the participants in CAWAPI was one to three orders of magnitude larger than that available for the NASA-TP calculations. The codes used in AVT-113 all ran on distributed memory parallelism, with the exception of the NLR code which exploits a very high vector performance. The cost of computing has fallen to such an extent that many participants used local resources, whereas Lamar $e t a l^{1}$ relied on an allocation on a supercomputer. 
Geometry Handling Emphasis in the working group was placed on retaining as much of the detailed definition of the aircraft as possible. A number of minor simplifications were made ${ }^{24}$ but to a very large degree this goal was achieved. A number of partners were able to use their own grid generation tools to generate a grid around the extremely complex shape. It seems likely that the geometry simplifications in Lamar et al. ${ }^{1}$ were driven by the need to keep the number of grid points down.

Grid Generation Several CAWAPI partners used their own tools to generate grids around the complex F-16XL shape. The most direct comparison with Lamar et al. ${ }^{1}$ is with the structured grid generation of NLR. It is clear that the tools developed at NLR represent a major advance on what was available at the time of Lamar et al. ${ }^{1}$ A significant development is the ability to generate unstructured grids for viscous flows through the exploitation of grown layers in the boundary layer. A number of codes were able to generate grids in a reasonable time. Finally, EADS-MAS and KTH/FOI made effective use of automatic grid adaption, the use of which is not currently as widespread as might have been expected.

Turbulence All participants in CAWAPI used turbulence treatments based on PDE's, in contrast to the algebraic model used in Lamar et al. ${ }^{1}$ The simplest turbulence treatment used was one or two-equation turbulence models. Rotation corrections to Boussinesq based models seemed to allow good solutions without too much difficulty, to the extent that they could be described as routine. Some partners used Reynolds stress models, but without significant obvious benefit. Finally, some partners used DES.

Unsteadiness Some partners showed it is now possible to resolve unsteady effects, showing significant unsteadiness downstream of the crank. The origins of this need further study, but could originate from an interaction of the inner and outer wing vortices, or an interaction between the inner wing vortex and the air dam.

Solver Discretization and solution schemes have advanced less since Lamar et al. ${ }^{1}$ The efficiency of the schemes was not really considered in CAWAPI, but interesting information about the performance on grids required for such a complex geometry could be obtained in the future. The spatial accuracy of the codes has not improved noticeably since the time of Lamar et al. ${ }^{1}$

Visualization Possibly driven by improved computing, the visualization of solutions produced in CAWAPI far exceeded that shown in the NASA-TP. Visualization through iso-surfaces, surface streamlines and the automatic detection of vortex cores all effectively 
showed the behavior of the solutions, including unsteady effects.

\section{B. Technology Readiness Level Improvements}

The following statements were made by industry team members of CAWAPI regarding the benefits accrued by their participating organizations in CAWAPI. Obviously, it is this group that will be end users of any new technology developed and, in that role the concept of TRL improvement is not only academic but economic, since company growth or survival can be an outcome. (The statements are arranged in the order received.)

\section{Mr. Willy Fritz of EADS-MAS, Munich Germany; comments}

Within the CAWAPI, EADS made essential experience with the solution based grid adaptation at complex configurations. We saw the benefit of the solution based grid adaptation, but we also learned that it is sometimes a laborious task. For the future application of the grid adaptation we got very useful guidelines.

Our daily business is not only to calculate force and moment coefficients or pressure distributions, but also to find out the physical causes for unexpected effects which were observed during flight test. Therefore we need a realistic computational model with correct slat-, flap- and rudder settings. So we have to use hybrid grids. These unexpected effects occur at the high angle of attack range or at special maneuvers. For these flow conditions, a carefully generated grid is an essential key towards a realistic numerical outcome. As grid-independent RANS solutions with globally refined hybrid grids are never possible in our production environment, the only possibility to minimize the grid dependency of the numerical outcome is again the use of solution adapted grids. Especially at vortical flow structures at high angles of attack even small vortices coming from slat corners, strakelets etc. can become very important, as they can trigger or delay vortex breakdown at the big primary vortices. Even in apparent very fine grids such small vortices are represented too weak.

The solution based grid adaptation very often tracks such small vortices and we get considerable improvements of the numerical outcome. Since the CAWAPI, we know that the solution based grid adaptation at such complex configurations can be handled and we know how to handle it. By this, the experience of CAWAPI has improved the readiness level of the application of our CFD-tools. (Without this experience we never would have tried a solution based grid adaptation at a complex configuration). 
CAWAPI was also the first possibility to validate our CFD-tools at a full scale fighter-type aircraft with flight test pressure distributions. (Agreements of the force and moment coefficients can be accidental as disagreements in the pressure distributions can cancel out each other).

Dr. Bruce Davis of Lockheed Martin Aeronautics, Ft. Worth, TX; comments

Lockheed Martin Aeronautics Company (LM Aero) as a designer, developer, and manufacturer of advanced military aircraft is keenly aware of the importance of accurate CFD simulations. CFD is used in a variety of ways, from improving aerodynamic design to providing aerodynamic loads to improving the separation of stores from aircraft. The validation of CFD codes is of central importance since this increases the confidence in CFD results and can potentially save costly wind tunnel and flight test time.

LM Aero has recently been involved in updating Falcon, its general purpose Navier-Stokes flow solver. This effort, and specifically the validation of the flow solver, has coincided with the involvement in the CAWAPI program. This timely involvement has provided LM Aero with a challenging validation suite with highquality flight test data. If for no other reason, the availability of this validation case has made the participation in CAWAPI a valuable experience for LM Aero, and the validation of our flow codes against this test database will not end with the CAWAPI program.

LM Aero did not generate the computational grids for this problem, but rather used grids supplied by the University of Tennessee Simulation Center (UTSimC). The grid used did not fully resolve the viscous sublayer of the turbulent boundary layer, but rather used wall functions. The LM Aero simulations underpredicted the suction peaks lying under the main vortices consistently. The suspicion is that the use of wall functions for this particular problem is not appropriate, and that simulations with the fully resolved viscous sublayer will bear this out. Even with the use of wall functions, the half-symmetry grid contained 42 million cells, which is the largest grid used to date for a Falcon v4 simulation. In this sense it allowed LM Aero to evaluate the code, as well as various utilities, for very large problems.

Dr. Todd Michal of Boeing Phantom Works, St. Louis, MO; comments

Participation in the CAWAPI program has provided many tangible and intangible benefits to Boeing. The CAWAPI collaboration has provided an opportunity 
to establish contacts with leading researchers while working toward the common goal of expanding the envelope of CFD analysis. Perhaps the most beneficial aspect of this program has been the establishment of best-practices for CFD analysis for complex high angle of attack, vortex dominated flow fields. Through our involvement, guidelines for grid properties, turbulence model, and algorithm option selection have been established that will directly benefit future Boeing programs. Another benefit of the CAWAPI effort has been the chance to compare and validate Boeing tools and processes with other industry, university and government codes. The comprehensive set of flight test results available for this effort enabled CFD to be validated in new parts of the flight envelope. This validation highlighted several areas where CFD tools do very well, and perhaps equally important, areas where current CFD methods fall short. It is our belief that identification of these short comings will guide future research efforts that will benefit Boeing as well as the greater CFD community.

In addition to benchmarking our current CFD capability, the CAWAPI program provided an excellent opportunity to investigate the use of new technologies to help solve complex problems. Capabilities such as grid adaptation, new turbulence models, and new numerical algorithms were all investigated. These studies provided a great deal of information that will help with the development and deployment of these new technologies.

\section{Conclusions}

Although differences were observed in the comparison of results from ten different CFD solvers with measurements, these solvers all functioned robustly on an actual aircraft at flight conditions with sufficient agreement among them to conclude that the overall objectives of the CAWAPI endeavor have been achieved. In particular, the status of CFD as a tool for understanding flight test observations has been confirmed. A number of specific points have arisen from CAWAPI:

- The "state-of-the-art" computations for FC70 showed a remarkable degree of agreement. However, the agreement with measurements was not close. After the assessment of several possible explanations it is suggested that this discrepancy is due to the poor prediction of a concentrated leading edge vortex and its interaction with a shock. This conclusion would not have been reached without performing a high-resolution inviscid analysis. These computations were only performed after the discrepancies between the "state-of-the-art" tools and measured data could not be explained. If this were 
an a-priori computational campaign, the conventional computations showing such a consistent agreement with each other, would have been interpreted differently. Then only a later flight test campaign would have recognized the discrepancy. This stands in clear contrast to the computations at FC25, where thanks to statistical tools the discrepancy between various computations would have triggered an alert. The "stateof-the-art" computations for FC70 would not have triggered such an alert. As shown at this condition, a necessary advance of the CFD tools is the reduction of discretization error, either from massive feature-driven grid adaption or higher order schemes.

- Time accurate simulations of the high incidence FC25 showed significant unsteadiness arising from the interactions of vortices downstream of the crank. This was unanticipated at the outset of the working group from a consideration of the flight test data. In this respect the CFD solutions have stimulated a reinterpretation of the measurements.

- Two considerations caused concern about the grid convergence of the CFD solutions. First, there is a large spread of the solutions for properties like vortex strength, as indicated by suction footprints on the wing surface. Secondly, grid adaption studies by some partners showed significant improvements in, for example, resolving secondary vortices. Given the large grids used (10-20 million points for half bodies), this emphasizes that advances in adaption and discretization scheme accuracy are important.

- The evaluation of predictions of boundary layer profiles showed the advantages of DDES over DES in this region.

- The generation of a block structured in similar times to those required to obtain quality unstructured grids showed what can be achieved with advanced grid generation tools generated by one of the CAWAPI partners.

The work of Lamar et al. ${ }^{1}$ provides a suitable benchmark for identifying the advances since 2001. This reference described an application of CFD to the F-16XL using state of the art tools at the time. The ability in the current working group to use grids of much higher resolution has brought the predictions more in line with measurements. In addition, advances in block structured grid generation tools have allowed the retention of almost all of the geometrical features in structured grids. Turbulence treatments which overcome the limitations of the Boussinesq assumption for vortical flow were used routinely. Unsteady effects were simulated by some partners and were shown to be significant. The accuracy of the spatial discretization has not advanced since 2001 and this has been shown to be significant. To balance this, some advances have been shown in grid adaption for unstructured codes. 


\section{Acknowledgment}

The authors gratefully acknowledge the support provided by Lockheed Martin Aeronautics Company - Fort Worth in providing a refined IGES geometry file and the parameter values of a generic engine that were subsequently used by facet members in their CFD studies, and the geometrical work performed by Mr. Edward B. Parlette of Vigyan, Inc. in generating a series of unstructured, tetrahedral grids from the IGES file, with the last one known as the base grid. Special thanks also to Todd Michal of Boeing, Stefan Goertz of DLR, David McDaniels of USAFA and Steve Karman of UT Chattanooga for their valuable suggestions and kind assistance in the writing of the paper.

\section{Appendix A. Statistical Analysis}

The forces and moments are statistically evaluated. The use of a statistical approach to analyze collective data is not new, ${ }^{25}$ the sequence of AIAA Drag Prediction Workshops used the approach adopted here. The statistical formulas used here are taken from Morisson and Hemsch. ${ }^{25}$ The basic idea is to analyze statistically the collective data to evaluate a mean and variance of the solution set and to identify those results which are statistically the same and those which are outliers, i.e. outside of the standard deviation. The population mean $\hat{\mu}$ is estimated using the sample median $\tilde{x}$, which is given (for the sorted data) as:

$$
\begin{aligned}
& \hat{\mu}=\tilde{x} \\
& \tilde{x} \equiv \begin{cases}x_{(n+1) / 2} & \text { if } n \text { is odd } \\
\frac{1}{2}\left(x_{(n / 2)}+x_{(n / 2)+1}\right) & \text { if } n \text { is even }\end{cases}
\end{aligned}
$$

The sample standard deviation $\hat{\sigma}$ is defined as

$$
\hat{\sigma}=\sqrt{\frac{1}{n-1} \sum x_{i}-\tilde{x}^{2}} .
$$

The upper and lower limits for a particular integrated variable (e.g. lift coefficient) define the boundaries for the outliers, they are:

$$
\begin{aligned}
\text { Upper limit } & =\hat{\mu}+k \hat{\sigma}, \\
\text { lower limit } & =\hat{\mu}-k \hat{\sigma}, \\
k & =\sqrt{3} .
\end{aligned}
$$




\section{References}

${ }^{1}$ Lamar, J. E., Obara, C. J., Fisher, B. D., and Fisher, D. F., "Flight, Wind-Tunnel, and Computational Fluid Dynamics Comparison for Cranked Arrow Wing (F-16XL-1) at Subsonic and Transonic Speeds," NASA/TP 2001-210629, NASA, February 2001.

${ }^{2}$ Obara, C. J. and Lamar, J. E., "Overview of the Cranked-Arrow Wing Aerodynamics Project International," Journal of Aircraft, Vol. -, No. -, - -, pp. -.

${ }^{3}$ Boelens, O. J., Badcock, K. J., Görtz, S., Morton, S. A., Fritz, W., Jr., S. L. K., Michal, T., and Lamar, J. E., "Description of the F-16XL Geometry and Computational Grids Used in CAWAP," Journal of Aircraft, Vol. -, No. -, --, pp.--.

${ }^{4}$ Boelens, O. J., Badcock, K. J., O.J., Elmilgui, A., K.S.Abdol-Hamid, and S.J.Massey, "Comparison of Measured and Block Structured Simulations for the F-16XL Aircraft," Journal of Aircraft, Vol. -, No. -, ,-- pp. - .

${ }^{5}$ Görtz, S., Jirásek, A., Morton, S. A., McDaniel, D. R., Cummings, R. M., Lamar, J. E., and AbdolHamid, K. S., "Standard Unstructured Grid Solutions for CAWAPI F-16XL," Journal of Aircraft, Vol. -, No. -, - -, pp. -

${ }^{6}$ Fritz, W., Davis, M. B., Karman, S. L., and Michal, T., "RANS Solutions for the CAWAPI F-16XL Using Different Hybrid Grids," Journal of Aircraft, Vol. -, No. -, - -, pp. -.

${ }^{7}$ Boelens, O. J., Spekreijse, S. P., Sytsma, H. A., and de Cock, K. M. J., "Comparison of measured and simulated flow features for the full-scale F-16XL aircraft," Proceedings of the 45th AIAA Aerospace Sciences Meeting \&3 Exhibit, Reno, NV, January 2007, AIAA Paper 2007-0489.

${ }^{8}$ Michal, T., Oser, M., Mani, M., and Ross, F., "BCFD Unstructured-Grid Predictions On The F16XL (CAWAPI) Aircraft," Proceedings of the 45th AIAA Aerospace Sciences Meeting 8 Exhibit, Reno, NV, January 2007, AIAA Paper 2007-0679.

${ }^{9}$ Badcock, K. J., "Evaluation of Results from a Reynolds Averaged Multiblock Code Against F-16XL Flight Data," Proceedings of the 45th AIAA Aerospace Sciences Meeting \& Exhibit, Reno, NV, January 2007, AIAA Paper 2007-0490.

${ }^{10}$ Elmiligue, A. A., Abdol-Hamid, K. S., and Massey, S. J., "PAB3D Simulations for the CAWAPI F-16XL," Proceedings of the 45th AIAA Aerospace Sciences Meeting \& Exhibit, Reno, NV, January 2007, AIAA Paper 2007-0491.

${ }^{11}$ Goertz, S. and Jirásek, A., "Unstructured Steady/Unsteady Solutions with Edge for CAWAPI F-16XL at KTH/FOI," Proceedings of the 45th AIAA Aerospace Sciences Meeting \& Exhibit, Reno, NV, January 2007, AIAA Paper 2007-0678.

${ }^{12}$ Fritz, W., "Hybrid Grid RANS Solutions For The CAWAPI F-16XL," Proceedings of the 45th AIAA Aerospace Sciences Meeting \& Exhibit, Reno, NV, January 2007, AIAA Paper 2007-0492.

${ }^{13}$ Karman, S., Mitchell, B., and Sawyer, S., "Unstructured Grid Solutions of CAWAPI F-16XL by UT SimCenter," Proceedings of the 45th AIAA Aerospace Sciences Meeting $\&$ Exhibit, Reno, NV, January 2007, AIAA Paper 2007-0681.

${ }^{14}$ Lamar, J. E. and Abdol-Hamid, K. S., "USM3D Unstructured Grid Solutions for CAWAPI at NASA 
LaRC," Proceedings of the 45th AIAA Aerospace Sciences Meeting 83 Exhibit, Reno, NV, January 2007, AIAA Paper 2007-0682.

${ }^{15}$ Morton., S. A., McDaniels, D. R., and Cummings, R. M., "F-16XL Unsteady Simulations for the CAWAPI Facet of RTO Task Group AVT-113," Proceedings of the 45th AIAA Aerospace Sciences Meeting E Exhibit, Reno, NV, January 2007, AIAA Paper 2007-0493.

${ }^{16}$ Davis, M. B., Reed, C., and Yagle, P., "Hybrid Grid Solutions on the (CAWAPI) F-16XL Using Falcon v4." Proceedings of the 45th AIAA Aerospace Sciences Meeting 83 Exhibit, Reno, NV, January 2007, AIAA Paper 2007-0680.

${ }^{17}$ Obara, C. J. and Lamar, J. E., "Overview of the Cranked-Arrow Wing Aerodynamics Project International," Journal of Aircraft, Vol. -, No. -, --, pp. -.

${ }^{18}$ Lamar, J. E. and Obara, C. J., "Review of Cranked-Arrow Wing Aerodynamics Project: Its International Aeronautical Community Role," Proceedings of the 45th AIAA Aerospace Sciences Meeting E Exhibit, Reno, NV, January 2007, AIAA Paper 2007-0487.

${ }^{19}$ Boelens, O. J., "Feature-based code validation using F-16XL flight test and wind tunnel data," NATO Research and Technology Organisation, Athens, Greece, October 2007, RTO-MP-AVT-147, chapter 56.

${ }^{20}$ Görtz, S., Jirásek, A., Morton, S. A., McDaniel, D. R., Cummings, R. M., Lamar, J. E., and AbdolHamid, K. S., "Standard Unstructured Grid Solutions for CAWAPI F-16XL," Journal of Aircraft, Vol. -, No. -, - -, pp. - .

${ }^{21}$ Cummings, R. M., Morton, S. A., Siegel, S. A., and Bosscher, S., "Numerical Prediction and Wind Tunnel Experiment for a Pitching Unmanned Combat Air Vehicle," Proceedings of the 41st Aerospace Sciences Meeting \& Exhibit, Reno, NV, January 2003, AIAA Paper 2003-0417.

${ }^{22}$ Jirásek, A. and Rizzi, A., "Numerical solution for the CAWAPI configuration on unstructured grids at KTH/FOI - Part II," NATO Research and Technology Organisation, 2007, RTO-TR-AVT-113, chapter 11.

${ }^{23}$ Le Moigne, Y., Adaptive Mesh Refinement and Simulations of Unsteady Delta-Wing Aerodynamics, Ph.D. thesis, KTH Farkost och flygteknik, 2004, ISBN 91-7283-770-3, TRITA-AVE 2004:17.

${ }^{24}$ Boelens, O. J., Goertz, S., Morton, S., Fritz, W., and Lamar, J. E., "Description of the F-16XL Geometry and Computational Grids Used in CAWAPI," Proceedings of the 45th AIAA Aerospace Sciences Meeting $\mathcal{G}$ Exhibit, Reno, NV, January 2007, AIAA Paper 2007-0488.

${ }^{25}$ Morrison, J. H. and Hemsch, M. J., "Statistical Analysis of CFD Solutions from the Third AIAA Drag Prediction Workshop (Invited)," Proceedings of the 45th AIAA Aerospace Sciences Meeting \&3 Exhibit, Reno, NV, January 2007, AIAA Paper 2007-0254. 